\title{
INSTABILITIES OF RELATIVISTIC STARS
}

\author{
K. D. KOKKOTAS AND J. RUOFF \\ Department of Physics, Aristotle University of Thessaloniki, Thessaloniki 54124, \\ Greece \\ E-mail: kokkotas@astro.auth.gr; ruoff@astro.auth.gr
}

\begin{abstract}
Recent developments on the rotational instabilities of relativistic stars are reviewed. The article provides an account of the theory of stellar instabilities with emphasis on the rotational ones. Special attention is being paid to the study of these instabilities in the general relativistic regime. Issues such as the existence relativistic r-modes, the existence of a continuous spectrum and the CFS instability of the w-modes are discussed in the second half of the article.
\end{abstract}

\section{Introduction}

The oscillations and instabilities of relativistic stars gained a lot of interest in the last decades because of the possible detection of their associated gravitational waves. It is not impossible that every compact star in a specific period of its life will undergo an oscillatory phase, in which it becomes unstable. Only the dynamical instabilities were thought to be a relevant source of gravitational waves, whereas instabilities due to dissipation mechanisms were believed to be only of academic interest. This belief has been dramatically altered during the last five years after it was discovered that for a specific class of rotational perturbations, the so called r-moded 1 , the instability due to gravitational radiation 3 . 6 has the potential of being a prime source for gravitational waves. It was subsequently shown that this instability has many interesting astrophysical implications, which attracted the attention of both relativists and astrophysicists.

In the first section of this review we shortly describe the various stellar instabilities and how they operate, for a more detailed review one can refer to a recent article by Stergioulast. The second section is devoted to the r-mode instability with the focus on its application to gravitational-wave research and astrophysics, for more details see a recent review by Andersson and Kokkotas 8 . The last section is devoted to an analysis of new features brought about by the relativistic treatment of the problem. Questions on the existence relativistic r-modes and a continuous spectrum are discussed in detail. A new result that is presented is the existence of the w-mode instability in ultra-compact stars, i.e. the instability due to the existence of an ergosphere of the spacetime or w-modese. 


\section{Stability of Relativistic Stars}

\subsection{Non-rotating stars}

There are many different types of instabilities which can operate in a relativistic star. The most familiar instability is due to the existence of a maximum mass beyond which the star collapses. In other words it becomes unstable with respect to radial perturbations. The points of instability can be easily found by constructing a sequence of stellar models for a particular equation of state (EOS), parametrized for example by the central density $\epsilon_{c}$. One can thus obtain the Mass-Radius diagram, where the extrema signal the transition from stability to instability and vice-versa. Within a perturbative approach, the stability analysis with respect to radial pulsations is reduced to a secondorder Sturm-Liouville type equation 10.11 with eigenvalue $\sigma^{2}$. For $\sigma^{2}>0$ the eigenfrequency $\sigma$ is real and the mode is stable while for $\sigma^{2}<0$ the eigenfrequency $\sigma$ becomes imaginary and the mode is unstable. The case $\sigma^{2}=0$ marks the onset of the instability. For the special case of uniform density stars, general relativity predicts that the star becomes unstable with respect to collapse if $M / R>8 / 9$.

For non-radial perturbations of non-rotating stars a standard criterion for mode stability is the sign of the so called Schwarschild discriminant

$$
\mathcal{A}_{s}=\frac{d p}{d r}-\left(\frac{\partial p}{\partial \epsilon}\right)_{s} \frac{d \epsilon}{d r}
$$

where $r$ is the radial coordinate, $p$ the pressure of the fluid and $\epsilon$ the total energy density. The condition $\mathcal{A}_{s}>0$ everywhere inside the star is necessary for stability. If $\mathcal{A}_{s}<0$ somewhere inside the star, the $g$-modes become unstable with respect to convection 12 . The stability of non-radially oscillating stars can also be studied via perturbation theory. One cay derive a system of perturbation equations for the fluid and the spacetime 13 . For relativistic stars the frequency of oscillation modes is no longer real valued because the non-radial oscillations generate gravitational radiation which dissipates energy from the star. Instead the frequency is complex $\left(\sigma=\sigma_{R}+i s i g m a_{I}\right)$ and the imaginary part of the frequency describes the damping or the growing

time of a mode. Unstable modes are characterized by $\tau<0$. For non-rotating relativistic stars with $\mathcal{A}_{s}>0$ no unstable modes have been found, although a rigorous proof of stability is still lacking.

florence: submitted to World Scientific on June 8, 2018 


\subsection{Rotating Stars}

The size of a rotating configuration is limited by the condition that the centrifugal acceleration should not exceed the centripetal acceleration of gravity. This leads to the following upper limit for the angular felocity $\Omega$ in terms of the mean density $\bar{\epsilon}$, established already a century ago 14 :

$$
\Omega^{2} \leq 2 \pi G \bar{\epsilon} .
$$

The limiting rotation frequency, $\Omega_{K}$, is called the Kepler limit. Although derived in Newtonian theory using simplifying assumptions, this relation is approximately correct for relativistic systems as wel 15,16 .

For uniformly rotating stars, stability against axisymmetric perturbations can be determined, via turning points along a constant angular momentum sequence 17 . An initially uniformly rotating relativistic star under the influence of such an axisymmetric instability becomes differentially rotating. Since the viscosity is the main mechanism for the redistribution of angular momentum within the fluid, the timescale for the development of the instability is set by viscous dissipation.

Rotational instabilities of stars arise from non-axisymmetric perturbations of the form $e^{i m \phi}$, where $\phi$ is the azimuthal angle. The bar mode $(m=2)$ is known to be the fastest growing unstable mode. There are two types of rotational instabilities: the dynamical and the secular ones.

- The dynamical instability is driven by hydrodynamical and gravitational forces and develops on an extremely short timescale, the dynamical timescale. For neutron stars it is of the order of milliseconds. Of special interest is the so called bar mode instability $(l=m=2)$ which deforms the star into in a bar shaped structure within approximately one rotational period. The bar mode instability is an extremely efficient mechanism of producing gravitational waves 18.29 .20

- The secular instabilities are driven by dissipative processes such as viscosity or gravitational radiation. They develop on much longer timescales, which are of the order of the dissipation time (for example the time required for viscosity to redistribute the angular momentum), i.e. tenths of seconds or even more.

The viscosity driven instability plays hardly any role in neutron stars because it occurs only in stellar models with much stiffer EOS than those considered to be realistid21.22.

Chandrasekhar 3 G first proved that gravitational radiation can drive Maclaurin spheroids, that are rigidly rotating uniform density spheroids, 
unstable. This instability causes the Maclaurin spheroids to evolve into a stationary but non-axisymmetric configuration, the Dedekind ellipsoide3. This interesting result was put on a rigorous footing by Friedman and Schutz: 24, who also proved that this instability is generic and that all rotating perfect fluid stars are unstable with respect to gravitational radiation.

\subsection{The CFS instability}

The mechanism for the gravitational-wave instability can be understood in the following way 6 . Consider first a non-rotating star. Then the modeproblem leads to eigenvalues for $\sigma^{2}$, i.e. one obtains equal values $\pm|\sigma|$ for the forward and backward propagating modes (corresponding to $m= \pm|m|$ ). These two mode branches are affected by rotation in different ways. The retrograde mode will be dragged forward by the stellar rotation. If the star spins sufficiently fast, this mode will appear moving forward in the inertial frame, but still backward in the rotating frame. Thus, an observer at infinity sees gravitational waves with positive angular momentum emitted by this retrograde mode, but since the perturbed fluid rotates slower than it would in absence of the perturbation, the angular momentum of the mode itself is negative. The emission of gravitational waves consequently makes the angular momentum of the mode increasingly negative thus leading to an instability.

From the above, one can easily conclude that a mode will be unstable if it is retrograde in the rotating frame (an observer rotating with the star and observing a frequency $\sigma$ for the mode) and prograde for a distant observer measuring a mode frequency $\sigma-m \Omega$, i.e. if

$$
\sigma(\sigma-m \Omega)<0
$$

For the high frequency $(f$ and $p)$ modes this is possible only for extremely high values of $\Omega$ or for quite large $m$. In general, for every mode there will always be a specific value of $m$ for which the mode will become unstable.

This class of frame-dragging instabilities is usually referred to as Chandrasekhar-Friedman-Schutz (CFS) instabilities. It is easy to see that the CFS mechanism is not unique to gravitational radiation. Any radiative mechanism will have the same effect.

A new result described in Section 4.3 is that the CFS mechanism is not only active for fluid modes but also for the spacetime or the so called $w$-modes 6 .

florence: submitted to World Scientific on June 8, 2018 


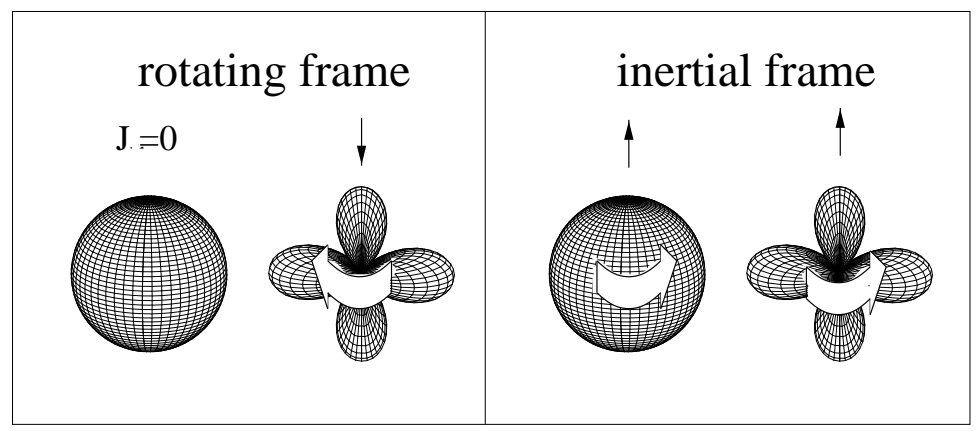

Figure 1. A schematic illustration of the conditions under which the CFS instability is operating. A perturbed star can be viewed as a superposition of a uniformly rotating background and a non-axisymmetric perturbation. A mode is unstable if it is retrograde according to an observer rotating with the fluid (left), but appears prograde for an observer at infinity (right).

\subsection{Dissipative Effects}

Dissipation is important for the stability/instability of rotating stars. In general dissipation due to the fluid viscosity tends to suppress the instability and thus stabilizes stars that do not rotate fast enough. The typical fluid viscosities that have been used for the study of unstable $f$ and $r$-modes are the shear and bulk viscosity 5 . The effects of the dissipation on the evolution of a perturbation are most easily studied by considering the energy of the perturbation as measured in the rotating frame

$$
E=\frac{1}{2} \int\left[\rho \delta u^{\alpha} \delta u_{\alpha}^{*}+\frac{\delta p \delta \epsilon^{*}}{\epsilon}+\delta \Phi \delta \epsilon^{*}\right] d V
$$

where $\delta u$ and $\delta \Phi$ are the perturbations of the fluid velocity and gravitational potential. In the absence of dissipation the energy of the mode is conserved. For a mode which is subject to dissipation, the eigenfunction is $\sim \exp [i(\sigma+i / \tau) t]$, where $\sigma$ is the real part of the mode frequency and $1 / \tau$ its imaginary part. The problem of estimating the stability of a mode is reduced to determining the sign of the imaginary part of the frequency. The imaginary part of the frequency is the ratio between the energy of the mode and the rate at which a specific dissipation mechanism dissipates energy from the mode, 
i.e.

$$
\frac{1}{\tau}=-\frac{1}{2 E} \frac{d E}{d t} .
$$

One now has to compare the damping/growth times due to the various mechanisms for energy dissipation in a given neutron star model

$$
\frac{1}{\tau}=\frac{1}{\tau_{\mathrm{gw}}}+\frac{1}{\tau_{\text {shear }}}+\frac{1}{\tau_{\text {bulk }}} .
$$

Rough estimates of the the various viscous mechanisms are given below.

- Shear Viscosity is the main dissipation mechanism for low temperatures $\left(T \leq 10^{9} \mathrm{~K}\right)$ and it arises from the momentum transport due to neutronneutron scattering. The dissipative energy loss in the mode energy is given by

$$
\frac{d E}{d t}=2 \int \eta \delta \sigma^{\alpha \beta} \delta \sigma_{\alpha \beta}^{*} d V,
$$

where $\sigma_{\alpha \beta}$ is the shear tensor and the thermodynamic function $\eta$ represents the shear viscosity of the fluid. A typical value for neutron stars is 2627

$$
\eta=2 \times 10^{18} \epsilon_{15}^{9 / 4} T_{9}^{-2} \mathrm{~g} \mathrm{~cm}^{-1} \mathrm{~s}^{-1} .
$$

- Bulk Viscosity dominates at high temperatures $\left(T \geq 10^{9} \mathrm{~K}\right)$ when matter becomes transparent to neutrinos. As the oscillating fluid undergoes compression and expansion, the weak interaction requires a relatively long time to re-establish equilibrium. This creates a phase lag between density and pressure perturbations which results to a large bulk viscosity 26 . Typically the mode energy lost through bulk viscosity is radiated away by neutrinos. This energy loss can be estimated by:

$$
\frac{d E}{d t}=\int \zeta \delta \sigma \delta \sigma^{*} d V
$$

where $\delta \sigma$ is the expansion associated with the mode and $\zeta$ the coefficient of the bulk viscosity, which for mode frequencies (in the rotating frame) has the value

$$
\zeta=6 \times 10^{25}(\sigma+m \Omega)_{1 \mathrm{~Hz}}^{-2} \epsilon_{15}^{2} T_{9}^{6} \mathrm{~g} \mathrm{~cm}^{-1} \mathrm{~s}^{-1} .
$$


- Gravitational Radiation dissipates energy from an oscillating star at a rate estimated via the post-Newtonian multipole-formula28 as

$$
\frac{d E}{d t}=-\sigma(\sigma+m \Omega) \sum_{l=2}^{\infty} N_{l} \sigma^{2 l}\left(\left|\delta D_{l m}\right|^{2}+\left|\delta J_{l m}\right|^{2}\right),
$$

where

$$
N_{l}=\frac{4 \pi G}{c^{2 l+1}} \frac{(l+1)(l+2)}{l(l-1)[(2 l+1) ! !]^{2}} .
$$

The radiation emitted is the sum of the mass $\delta D_{l m}$ and current $\delta J_{l m}$ multipole contributions given by

$$
\begin{aligned}
\delta D_{l m} & =\int \delta \epsilon r^{l} Y_{l m}^{*} d V, \\
\delta J_{l m} & =\frac{2}{c}\left(\frac{l}{l+1}\right)^{1 / 2} \int r^{l}(\epsilon \delta \vec{u}+\vec{u} \delta \epsilon) \cdot \vec{Y}_{l m}^{B *} d V,
\end{aligned}
$$

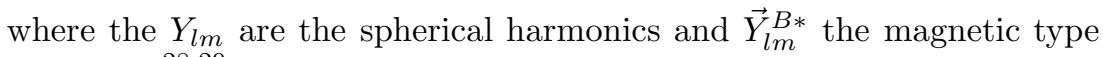
multipoles28229.

By estimating the energy of the mode using equation (4) and the energy dissipation via (7), (9) and (11) one gets all the components needed in equation (6). For a given EOS and a given stellar model the sign, which signals if the mode is growing or not, of equation (6) is a function of the rotational period and the temperature of the star. Figure 2 shows the instability window, obtained in this way, of the $l=m=2 r$-mode.

\section{$3 \quad r$-modes}

The oscillation patterns of a star are classified according to the dominant restoring force that tries to push a displaced fluid element back into its equilibrium position. The two main families of modes, which exist for any type of stars (rotating or not), are those whose restoring forces are pressure ( $p$ modes) and buoyancy ( $g$-modes). Rotation not only shifts the spectra of these modes but additionally gives rise to a new type of restoring force, the Coriolis force, with an associated new family of rotational modes. In general these are called inertial modes and are known in geophysics as Rossby waves. Of special interest to us is the quadrupole $r$-mode with $l=2, m=2$.

Inertial modes are primarily velocity perturbations. In the slow rotation limit, the velocity field of the $r$-mode is given by

$$
\delta \vec{u} \sim \alpha R \Omega\left(\frac{r}{R}\right)^{l} \vec{Y}_{l l}^{B} e^{i \sigma t},
$$


where $\alpha$ is the mode amplitude and $R$ the radius of the star. In a first approximation the fluid elements have no radial displacement and move on paths which are approximately ellipses with $\theta$-dependent eccentricities

$$
\frac{\xi_{\theta}^{2}}{\sin ^{2} \theta}+\frac{\xi_{\phi}^{2}}{\sin ^{2} \theta \cos ^{2} \theta} \sim \alpha^{2} R^{2},
$$

see for example Fig. 1 in 8 . The associated density perturbations are small for low rotation rates $\delta \rho \sim \mathcal{O}\left(\Omega^{2}\right)$ and at the Newtonian level, the mode frequency in the rotating frame of reference is

$$
\sigma=\frac{2 m \Omega}{l(l+1)} .
$$

Using the criterion for CFS stability given by equation (3) we get

$$
\sigma(\sigma-m \Omega)=-\frac{2(l-1)(l+2) m^{2} \Omega^{2}}{l^{2}(l+1)^{2}}<0,
$$

which implies that the $r$-mode is unstable for any rotation rate of the star. This was first pointed out 4 years agoll and immediately created considerable excitement because of the astrophysical implications this instability would have for the spin evolution of newly born neutron stars and for the search for gravitational waves.

The main gravitational wave output from $r$-modes is not due to disturbances in the star's fluid density (mass multipoles, $\delta D_{l m} \sim \Omega^{2}$ ) but originates from the time dependent mass currents (current multipoles, $\delta J_{l m} \sim \Omega$ ). This is the gravitational analogue of magnetic multipole radiation and is a rare case among the expected astrophysical sources of gravitational radiation.

\subsection{Instability window}

One can estimate the growth time of the instability using equation (5) together with the viscous damping times. In a first approximation one obtains

$$
\begin{aligned}
\tau_{\mathrm{gw}} & \sim 47 M_{1.4}^{-1} R_{10}^{-2 l} P_{-3}^{2 l+2} \mathrm{sec}, \\
\tau_{\mathrm{sv}} & \sim 7 \times 10^{7} M_{1.4}^{-5 / 4} R_{10}^{23 / 4} T_{9}^{2} \mathrm{sec} \\
\tau_{\mathrm{bv}} & \sim 3 \times 10^{11} M_{1.4} R_{10}^{-1} P_{-3}^{2} T_{9}^{-6} \text { sec. }
\end{aligned}
$$

From the above values one can easily show that for high temperatures $\left(T \geq 10^{10} \mathrm{~K}\right)$ the bulk viscosity suppresses any oscillations, while the shear viscosity dominates for temperatures below $10^{7} \mathrm{~K}$. Still, as shown in Fig. 2, for temperatures between $10^{7}$ and $10^{10}$, there is a broad window in which the instability can operate 29.30 .

florence: submitted to World Scientific on June 8, 2018 


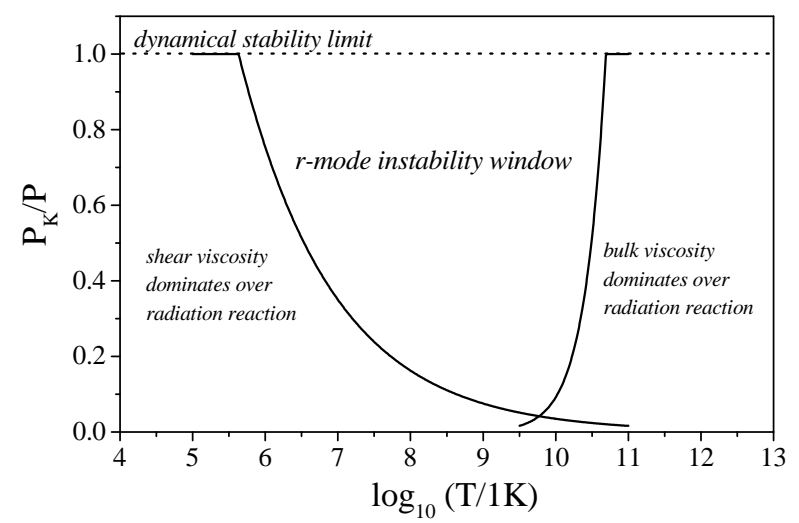

Figure 2. The instability window for the $l=m=2 r$-mode of a typical neutron star $\left(R=10 \mathrm{~km}\right.$ and $M=1.4 M_{\odot}$ and Kepler period $\left.P_{K} \approx 0.8 \mathrm{~ms}\right)$.

In particular these results imply that for temperatures of the order of $10^{9} \mathrm{~K}$ a small rotation rate of the order of $5 \%$ of the Kepler frequency $\Omega_{K}$ is already sufficient to drive the star unstable.

The growth time due to the mass multipole radiation of the r-modes is about four orders of magnitude longer and the corresponding instability window is considerably smallert. The picture is similar for $r$-modes with higher $l$.

In order to obtain a more realistic description of neutron stars, one should also take into account the effects of a solid crust. In old neutren stars a solid crust significantly affects the size of the instability window 13.32 .33 . 34 but has been suggested to have no effect for the evolution of young neutron stars. The size of the window is shown in Fig. 3, where we discuss the influence of the $r$-mode instability in accreting old neutron stars.

Strange stars, if they exist, might consist of the most stable known form of matter, which is a mixture of strange, up and down quarks. There are speculations that a considerable number of the observed compact stars fall into this category. Both shear and bulk viscosity are significantly different for strange stars, which has a significant influence on the instability window 35 . Bulk viscosity is dominant for temperatures between $10^{8}-10^{10} \mathrm{~K}$ and as a result the instability window is considerably smaller and moves to lower temperatures. As we will show later, this has a significant effect for Low-Mass X-Ray Bi- 
naries (LMXB)36. In addition a new instability window appears for higher frequencies, see Fig. 7 in $\mathrm{l}$.

In specific EOS it is expected that the central parts of the neutron star cores contain a significant number of hyperons. Jones 37.38 estimated that the bulk viscosity due to non-leptonic weak interactions involving hyperons will beconsiderably stronger. In a more detailed calculation Lindblom and Owen 39 have verified this result and have shown that the instability window is considerably smaller. They suggested that for young neutron stars, containing a considerable amount of hyperons in their cores, the r-mode instability will be suppressed before it significantly affects the angular momentum of the star.

\subsection{Evolution of nascent neutron stars}

Neutron stars are born after the gravitational collapse of the neutron-star progenitor (massive star or accreting white dwarf). Conservation of angular momentum suggests that they should rotate almost at break-up speed, i.e. near their Kepler limit. Moreover, they should be quite hot with temperatures of the order of $10^{11} \mathrm{~K}$. These nascent neutron stars cool down by neutrino emission within a few seconds to temperatures below $10^{10} \mathrm{~K}$ without considerable loss of angular momentum. According to Fig. 2 the star then enters the instability window and the amplitude of the $r$-mode starts to grow exponentially. After a few minutes, nonlinear effects take over and saturate the highly unstable mode. When saturation occurs the star enters a nearly steady state, in which the amplitude of the mode remains essentially unchanged and the angular momentum of the star is radiated away while it gradually cools down. After a year or so the angular velocity and the temperature of the star are low enough that viscous damping can start to dominate over the $r$-mode instability. Consequently, the star moves out of the instability window and the phase of angular momentum loss to gravitational radiation ends. The star keeps cooling down slowly while its angular velocity remains practically constant. In the case of the best known pulsar, the Crab pulsar, this evolutionary scenario has provided some impressive results. The Crab pulsar has an observed period of $33 \mathrm{~s}$, but 1000 years ago when it was born, it had an estimated period of $19 \mathrm{~s}$, which is exactly the period that the evolutionary model predicts: Initially born with a period of 1-2 ms, the Crab pulsar has radiated away about $95 \%$ of its angular momentum in the form of gravitational waves within a year, thereby slowing down to a period of $\sim 19 \mathrm{~s}$.

For young strange stars, it has been shown that the $r$-mode driven spinevolution is quite different from the neutron star case 36 . In a young strange star, the r-mode undergoes short cycles of instability during the first few 
months, followed by a quasi-adiabatic phase, where the $r$-mode remains at a small and roughly constant amplitude for thousands of years. Another important feature from the neutron-star case is that the $r$-mode in a strange star never grows to large amplitudes 36 . These results suggest that the $r$-modes in young strange stars emit a persistent gravitational-wave signal that should be detectable with large-scale interferometers, given an observation time of a few months 36 .

\subsection{Gravitational radiation emission}

The aforementioned $95 \%$ loss of angular momentum due to the $r$-mode instability is transformed into gravitational radiation, which is emitted at a frequency range of 50-1000 Hz. This large amount of angular momentum loss in gravitational waves makes the rotational instabilities one of the primary sources for the detection of gravitational waves with laser interferometric detectors.

The frequency of the waves produced by an $\bar{T}=2, m=-2 r$-mode is $f_{\mathrm{gw}}=2 \Omega / 3 \pi$ and the expected strain amplitude is 40

$$
h(t)=10^{-24} \alpha M_{1.4} R_{10}^{3}\left(\frac{15 \mathrm{Mpc}}{D}\right),
$$

where $D$ is the distance from the source. The signal to noise ratio for the above signal is

$$
\left(\frac{S}{N}\right)^{2}=2 \int_{0}^{\infty} \frac{\mid \tilde{h}(f)]^{2} d f}{S_{h}(f)}
$$

where $\tilde{h}(f)$ is the Fourier transform of the signal and $S_{h}(f)$ is the one-sided power spectral density of the detector noise. If the saturation amplitude is about unity, newly formed neutron stars out to about $10 \mathrm{Mpc}$ should be detectable by LIGO II with narrow banding. This suggests that the Virgo cluster is likely to be out of reach 11 . For the first phase of the instability, when the signal grows up to a saturation point, there are estimates predicting an upper signal to noise ratio of $S / N<10$ for the LIGO II configuration 42 .

\subsection{Accreting neutron stars}

The $r$-mode instability revived the interest in the gravitational radiation producing mechanism suggested originally by Papaloizou and Pringle 43 and later also by Wagoner 4 . The mechanism applies to old neutron stars which are spun up by accretion until they reach the instability window. For these stars

florence: submitted to World Scientific on June 8, 2018 
it was assumed that the loss of angular momentum via gravitational wave should balance the accretion torque.

This scenario, initially suggested for $f$-modes, has been revived for the $r$-modes 4 . 46 . It was soon realized 4 that the viscous heating of the neutron star will not allow the star to remain in the equilibrium state suggested by Wagoner. Instead, the runway reheating will drive the star further into the instability regime (path A-B in Fig. 3) and spin it down (path B-C). This process will last for a few months after which the star will follow the path $(\mathrm{C}-$ D-A), i.e. it will cool down and spin up again due to accretion. Eventually, it will reach the instability window again and repeat this cycle. This quiet phase lasts for about $10^{6}$ years, which means that an accreting neutron star spends only a tiny fraction of its accretion lifetime in the phase A-B-C, during which it emits gravitational radiation. Still, Fig. 3 shows three remarkable result.48. First the observed LMXB seem to follow the pattern suggested here, second this mechanism sets a lower limit for the rotation period of the recycled pulsars and third it provides an explanation for the period clustering of the millisecond pulsars. The point A in Fig. 3 corresponds to the smallest possible period that the pulsar can achieve via accretion. Remarkably, the theoretical value is $1.5 \mathrm{~ms}$ while the fastest known pulsar rotates with a period of $1.56 \mathrm{~ms}$. Pulsars following this accretion scenario should have periods bounded by the two points $\mathrm{A}$ and $\mathrm{D}$, which correspond to 1.5 and $6.5 \mathrm{~ms}$ and this is true for the majority of the known millisecond pulsars.

Although Wagoner's original scenario is not realized for normal neutron stars, it applies to strange stars. The instability window is quite different here and it has been found that unstable $r$-modes affect strange stars in a way that is quite distinct from the neutron star case. For accreting strange stars, the onset of the $r$-mode instability does not lead to the thermo-gravitational runaway that is likely to occur in neutron stars36. Instead, the strange star evolves towards a quasi-equilibrium state on a timescale of about a year. The reason is that the instability window moves towards lower temperatures and the corresponding point $\mathrm{A}$ is on the left side of the window. When the star becomes unstable, the runaway heating pushes the star to higher temperatures i.e. outside the window (ordinary neutron stars are pushed further inside). The star becomes stable again and soon cools down and "hits" the instability line again and the process continues for as long as the accretion lasts 36 . This is shown in Fig. 4. The upper panel shows the time evolution of the mode amplitude $\alpha$. After a few initial peaks, which are of the order of $\alpha \sim 10^{-4}$, the amplitude settles to a constant level of about $10^{-5}$. During this "stable" phase the mode heating remains also constant (middle panel) while the rotational period of the star increases because of the continuous loss of angular momen- 


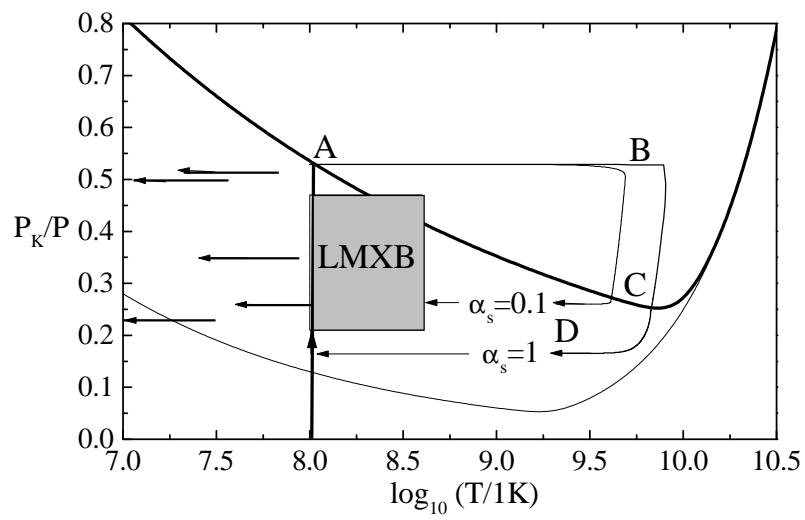

Figure 3. The $r$-mode instability window relevant for old neutron stars. We show results for the simplest (crust-free) model (thin solid line), as well as for a star with a crust (thick solid line). We illustrate two typical $r$-mode cycles (for mode saturation amplitudes $\alpha_{s}=0.1$ and 1), resulting from thermo-gravitational runaway after the onset of instability. For comparison with observational data, we indicate the possible range of spin-periods inferred from current LMXB data (shaded box) as well as the observed periods and estimated upper limits of the temperature of some of the most rapidly spinning millisecond pulsars (short arrows).

tum in gravitational waves. This mechanism could explain the clustering of spin-frequencies inferred from $\mathrm{kHz}$ QPO data in LMXB. These results suggest that the $r$-modes in strange stars emit a persistent gravitational-wave signal that should be detectable with large-scale interferometers given an observation time of a few months. If detected, these signals would provide unique evidence for the existence of strange stars, which would put useful constraints on the parameters of QCD.

\subsection{Magnetic Fields}

Neutron stars usually have strong magnetic fields, which should interact with a large amplitude pulsation mode. It has been suggested that a magnetic field should have a strong influence on the evolution of the $r$-mode instability and vice-versa. Spruit 49 suggested that the $r$-mode should generate some kind of differential rotation. In this case the magnetic field winds up until it reaches a critical point where it becomes unstable due to buoyancy forces. When this 


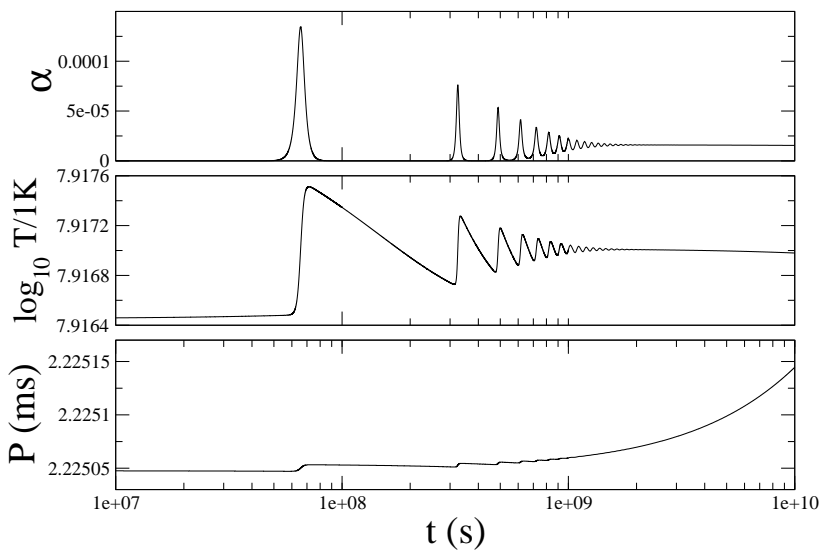

Figure 4. The evolution of the $r$-mode amplitude (upper frame), the temperature (middle frame) and the spin period (bottom frame) corresponding to the first $10^{10} \mathrm{~s}$ following the initial onset of $r$-mode instability in an accreting strange star.

happens the star emits a considerable amount of electromagnetic radiation and perhaps gamma rays. As a consequence the neutron star will acquire an extremely strong magnetic field and hecome a magnetar.

Rezzolla, Lamb and Shapiro50 5152 suggested that the magnetic field might actually prevent the $r$-mode instability at all. This is possible if the non-linear evolution of the $r$-modes winds up the magnetic field of the neutron star in such a way that it drains energy away from the mode and thus suppresses it entirely. For magnetic fields of the order of $B \geq 10^{10} \mathrm{G}$, the rotation rate must be above $0.35 \Omega_{K}$ in order for the mode to survive, while for stronger magnetic fields of the order of $B>10^{15} \mathrm{G}$, the $r$-mode cannot grow at all. Ho and Lai53 discussed the effect of magnetic braking on the $r$-mode growth and showed that it is important only for extremely strong magnetic fields $B \geq 10^{14} \mathrm{G}$. A more systematic study of the effect of the magnetic field on the $r$-modes was recently published by Morsink and Rezania 54 .

\subsection{Nonlinear Calculations}

Many of the results that we presented till now were based on the assumption that the $r$-modes reach an amplitude of order unity and then saturate due to nonlinear processes. The way that the nonlinear effects influence the saturation of a mode is not known and there are questions which need to be answered. For example, does the coupling of the $r$-mode to other modes allow 
the mode to grow to unit amplitude? Does the growth of the mode influence an initially uniformly rotating star in such a way that it becomes differentially rotating? We have seen that this is an important assumption for the influence of the magnetic field.

Nonlinear studies of pulsation modes suffer from the immense computer power needed and both approaches that we will discuss are limited by the computer resources. Stergioulas and Font 55 evolve a model of a rapidly rotating relativistic neutron star using the nonlinear hydrodynamical equations without evolving the spacetime (Cowling approximation). In this way one cannot see the actual growth of the mode, but by evolving a sequence of perturbed configurations with increasing initial mode amplitude $\alpha$, one can study at least the energy leak from the $r$-mode into other modes on a dynamical timescale. Though some other modes have been excited, no mode coupling has been observed at amplitudes of order unity at the resolution used in these simulations.

These results were verified by another nonlinear calculation by Lindblom, Tohline and Vallisneri565. They worked in Newtonian theory and used a post-Newtonian radiation-reaction force to drive the $r$-mode unstable. In order to shorten the growth time, they increased the driving radiation reaction force by a factor of 4500 . In this way, they could observe the growth of the mode from an amplitude $\alpha=0.1$ to $\alpha=2$. These results verified earlier approximate calculations 40 of the loss of angular momentum of the star. They have not observed significant mode coupling either but at an amplitude $a \sim 3.4$ they found nonlinear saturation, which they argue was due to the creation of shocks associated with the breaking of surface waves on the star. In both calculations it was observed that the $r$-modes generated differential rotation.

In two recent papers, Schenk at al 58 and Morsink 59 developed a formalism to study the nonlinear interaction of modes in rotating Newtonian stars. Subsequently they used this formalism to study the saturation amplitude of the $r$-mode instability 60 . Their results suggest that the saturation amplitude is extremely small and never reaches amplitudes of order unity. Instead they showed that the mode saturates at an amplitude which is abgut four orders of magnitude smaller than that suggested by Lindblom et al.56. This obvious disagreement maybe a consequence of the limited resolution in the nonlinear time evolutions 55 56.

Recent estimations by Wagonen 61 suggest, that even if one takes into account the assumption that the r-mode amplitude will never reach values of the order 1, the r-mode instability in accreting neutron stars will still be a very good source for gravitational waves. This result is similar to the one presented earlier by Andersson et al. 16 for accreting strange stars.

florence: submitted to World Scientific on June 8, 2018 


\section{Instabilities of rotating stars in GR}

The theory of non-radial perturbations of relativistic stars has been a field of intensive study for more than threedecades, beginning with the pioneering paper of Thorne and Campolattard 13 .

These authors focused on perturbations of non-rotating stars while Hartle 62 laid the foundations for computing rotating relativistic stellar models. He also devised a way of modelling slowly rotating stars. This slow-rotation approximation is still widely used since the problem becomes one dimensional and therefore much simpler than the two dimensional case of rapidly rotating and strongly deformed stars.

Inyestigating the axisymmetric perturbations, Chandrasekhar and Ferrari63 showed how rotation induces coupling of the polar and axial modes, which are decoupled in the non-rotating case. Soon after, Kojima64 presented the first complete derivation of the coupled polar and axial perturbation equations. Rotation does not only couple the polar and axial modes, it also removes the $(2 l+1)$-fold degeneracy of a mode with angular index $l$ in a non-rotating star. To first order, this Zeeman-like frequency splitting is linear in the rotation parameter. Kojima 65.60 computed the splitting for the relativistic $f$-mode for various sequences of polytropic models. Yoshida and Kojima 6 compared the frequencies and their rotational corrections for $f$ and $p$-modes in the relativistic Cowling approximation with the fully relativistic perturbation calculation. They concluded that the Cowling approximation is remarkably good and becomes better the more relativistic the stellar models are.

The "run" on the $r$-modes started, when Anderssont found that they are CFS-unstable for any rotation rate. In a first attempt to compute the growth time withip a fully relativistic framework, he used the axial equation derived by Kojima 64, without the coupling to the polar equations. Although he found the right sign of the imaginary part of the complex $r$-mode frequencies, he was not able to recover the $\Omega^{-(2 l+2)}$ behavior (see eqn(19)). Ruoff and Kokkotasi6 pointed out that Kojima's equation is not adequate for computing $r$-modes, for it is derived by transforming the original axial field equations, which are first order in time (or in the eigenfrequency $\sigma$ ), into a second order wave equation. In these manipulations, the eigenvalue $\sigma$ appears quadratically, and one obtains second order rotational corrections, which, however, were discarded. Since the $r$-mode frequency $\sigma$ is proportional to the rotation rate $\Omega$, it is clear that one has to keep the $\Omega^{2}$ terms in the wave equation.

Taking care of the proportionality between $\sigma$ and $\Omega$, Kojima 69 derived an approximate equation in the so-called low-frequency approximation, where

florence: submitted to World Scientific on June 8, 2018 
the gravitational radiation reaction is ignored. This equation is now an eigenvalue equation, which is linear in $\sigma$ and contains only rotational correction terms linear in $\Omega$. Kojima immediately realized that the relativistic treatment introduced a new effect, not present in the Newtonian case, namely the appearance of a continuous spectrum, arising from the relativistic frame dragging, which introduces an effective $r$-dependent oscillation frequency. The presence of this continuous spectrum in Kojima's equation has been proven in a mathematically rigorous way by Beyer and Kokkotas 7 .

For quite a while, it was not clear whether this continuous spectrum was merely an artefact of the low-frequency approximation, which neglects the radiation reaction, or whether it occurred because of the neglect of the coupling to the polar equations, or a feature of the slgw-rotation approximation in general. Lockitch, Andersson and Friedman 11 argued that Kojima's equation should only be valid for non-barotropic stars, and showed that for uniform density models, one can find regular mode solutions, representing the relativistic $r$-modes.

Ruoff and Kokkotas 12 and Yoshida 23 extended this study to polytropic models and found the somewhat surprising results that Kojima's equations yields $r$-mode solution only for a very restricted class of polytropic models, namely for those which small polytropic index $n$. For large $n$, no regular solutions could be found. Going one step further Ruoff and Kokkotas 67 and Yoshida and Futamase 4 included the radiation reaction, but still found that the basic properties of Kojima's equations do not change and its singular structure due to the continuous spectrum cannot be avoided.

Kojima and Hosonuma 75 showed that if third-order rotational effects are added to the original Kojima's equation, it becomes a fourth order ordinary differential equation, which has no singular properties if the Schwarzschild discriminant associated with the buoyant force does not vanish inside the star. By solving a simplified toy version of the extended Kojima's equation, Lockitch and Andersson 76 showed that the previously singular solutions can be regularized when the additional fourth-order term is taken into account.

Yoshida and Lee 77 and Ruoff, Stavridis and Kokkotas 78 considered the fully coupled system in the Cowling approximation by including only first order corrections. The difference to the previous investigations is that the computation of the mode frequencies and eigenfunctions is not done in a perturbative method, in which $\Omega$ is regarded as a small expansion parameter. Instead the mode is a solution of the complete coupled system, with $l$ ranging from $l=m$ to infinity. For the numerical computation, this system has to be truncated at some cut-off value $l_{\max }$ which has to be chosen such that the eigenfrequencies and eigenfunctions are well converged as $l_{\max }$ is increased. 
Yoshida and Lee 77 focused on non-barotropic models taken from McDermott, van Horn and Hansen 79 , which have a finite $r$-dependent temperature. For all models under consideration, they found regular $r$-mode solutions, and all of them were lying in the regime of the continuous spectrum, which was forbidden from Kojima's equation.

Ruoff et al. 88 considered barotropic as well as non-barotropic polytropic stellar models and demonstrated that in both cases, one can always find $r$ modes. They discussed how the truncation of the coupled system of equations at some cut-off value $l_{\max }$ affects the continuous spectrum, which for the coupled equations consists not only of a single band, but of several either disconnected or overlapping patches. They found that some of the inertial modes, in particular the $r$-mode, can actually exist inside the continuous spectrum. However, for highly relativistic stellar models, the continuous spectrum can still make some of the inertial modes, which exist in less relativistic stars, disappear.

In the remainder of this review, we will outline the various approximations and the results they are leading to. We will use geometrical units with $G=$ $c=1$.

\subsection{The slow-rotation formalism}

The metric describing a slowly rotating neutron star in spherical coordinates $(t, r, \theta, \phi)$ is

$$
g_{\mu \nu}=\left(\begin{array}{cccc}
-e^{2 \nu} & 0 & 0 & -\omega r^{2} \sin ^{2} \theta \\
0 & e^{2 \lambda} & 0 & 0 \\
0 & 0 & r^{2} & 0 \\
-\omega r^{2} \sin ^{2} \theta & 0 & 0 & r^{2} \sin ^{2} \theta
\end{array}\right),
$$

where $\nu, \lambda$ and the "frame dragging" $\omega$ are functions of the radial coordinate $r$ only. With the neutron star matter described by a perfect fluid with pressure $p$, energy density $\epsilon$, and 4 -velocity

$$
U^{\mu}=e^{-\nu}(1,0,0, \Omega),
$$

the Einstein equations together with an equation of state $p=p(\epsilon)$ yield the well known TOV equations plus an extra equation for the frame dragging, which to linear order is given by

$$
\varpi^{\prime \prime}-\left(4 \pi r e^{2 \lambda}(p+\epsilon)-\frac{4}{r}\right) \varpi^{\prime}-16 \pi e^{2 \lambda}(p+\epsilon) \varpi=0,
$$

where

$$
\varpi:=\Omega-\omega
$$


represents the angular velocity of the fluid relative to the local inertial frame.

We assume the oscillations to be adiabatic, so that the relation between the Eulerian pressure perturbation $\delta p$ and energy density perturbation $\delta \epsilon$ is given by

$$
\delta p=\frac{\Gamma_{1} p}{p+\epsilon} \delta \epsilon+p^{\prime} \xi^{r}\left(\frac{\Gamma_{1}}{\Gamma}-1\right),
$$

where $\Gamma_{1}$ represents the adiabatic index of the perturbed configuration, $\Gamma$ is the background adiabatic index

$$
\Gamma=\frac{p+\epsilon}{p} \frac{p^{\prime}}{\epsilon^{\prime}},
$$

and $\xi^{r}$ is the radial component of the fluid displacement vector $\xi^{\mu}$. If we write $\Gamma_{1}$ as

$$
\Gamma_{1}=\frac{p+\epsilon}{p}\left(\frac{\partial p}{\partial \epsilon}\right)_{s},
$$

we can write relation (28) as

$$
\delta p=\left(\frac{d p}{d \epsilon}\right)_{s} \delta \epsilon-\xi^{r} \mathcal{A}_{s}
$$

with the Schwarzschild discriminant $\mathcal{A}_{s}$ given by equation (1).

The metric perturbations $h_{\mu \nu}$ are functions of all four spacetime variables

$$
h_{\mu \nu}=h_{\mu \nu}(t, r, \theta, \phi)
$$

To eliminate the angular dependence, one expands the perturbations into tensorial harmonics, which are a generalization of the well-known spherical harmonics $Y_{l m}=Y_{l m}(\theta, \phi)$. One thus obtains a set of equations for the coefficients, which only depend on $t$ and $r$ and the parameters $l$ and $m$. In the non-rotating case, these equations are independent of $m$ and decoupled with respect to $l$. Furthermore, they fall into two independent sets, which show opposite behavior under parity transformation. The polar or even parity perturbations transform as $(-1)^{l}$, whereas the axial or even parity perturbations as $(-1)^{l+1}$. Rotation induces a coupling between the different sets, through first order rotational terms, which couple the set of polar/axial equations of order $l$ to the set of axial/polar equations of order $l \pm 1$. Furthermore, the equations now depend explicitly on $m$.

In deriving the perturbation equations, one has to make use of the wellknown gauge freedom. In the non-rotating case, the commonly used gauge is the Regge-Wheeler gauge. However, in the rotating case, it is more convenient 
to use a different gauge, the BCL gauge, which was first introduced by Battiston, Cazzola and Lucaronil 80 . A convenient way to set up this gauge and to derive the perturbation equations for slowly rotating relativistic stars is to use the ADM-formalism 81, 82. The ADM formalism provides a natural framework to derive the perturbation equations for rotating stars, as it clearly separates the equations into dynamical evolution equations and the set of time independent constraints. As a consequence the equations do not contain any mixed time and space derivatives and in the BCL gauge, they do not contain any second order derivatives at all. This makes them particular suitable for the numerical time evolution, and they can also be easily cast into an eigenvalue problem.

In the ADM formalism, the gauge is chosen by giving prescriptions for the lapse function $\alpha$ and the shift vector $\beta^{i}$. As we are dealing with perturbations, $\alpha$ and $\beta^{i}$ are the perturbations of lapse and shift. In the BCL-gauge, one sets $\alpha=0$ and $\beta^{i}$ is chosen such that the evolution equations for the angular perturbations $h_{\theta \theta}, h_{\theta \phi}$ and $h_{\phi \phi}$ become

$$
\partial_{t} h_{a b}=0, \quad a, b=\{\theta, \phi\} .
$$

With $h_{a b}=0$, the expansion of the metric perturbation is

$$
\begin{aligned}
\alpha & =0, \\
\beta_{i}^{\text {polar }} & =\sum_{l, m}\left(e^{2 \lambda} S_{2}^{l m}, V_{1}^{l m} \partial_{\theta}, V_{1}^{l m} \partial_{\phi}\right) Y_{l m}, \\
h_{i j}^{\text {polar }} & =\sum_{l, m}\left(\begin{array}{ccc}
e^{2 \lambda} S_{3}^{l m} & V_{3}^{l m} \partial_{\theta} & V_{3}^{l m} \partial_{\phi} \\
\star & 0 & 0 \\
\star & 0 & 0
\end{array}\right) Y_{l m},
\end{aligned}
$$

for the polar part and

$$
\begin{aligned}
\beta_{i}^{a x i a l} & =\sum_{l, m}\left(0,-V_{2}^{l m} \frac{\partial_{\phi}}{\sin \theta}, V_{2}^{l m} \sin \theta \partial_{\theta}\right) Y_{l m}, \\
h_{i j}^{a x i a l} & =\sum_{l, m}\left(\begin{array}{ccc}
0-V_{4}^{l m} \frac{\partial_{\phi}}{\sin \theta} V_{4}^{l m} \sin \theta \partial_{\theta} \\
\star & 0 & 0 \\
\star & 0 & 0
\end{array}\right) Y_{l m} .
\end{aligned}
$$

for the axial part. The asterisks stand for symmetric components. The perturbations of the extrinsic curvature are all non-zero and denoted by $K_{1}$ through $K_{6}$. Of these, $K_{3}$ and $K_{6}$ represent the axial perturbations. Finally, the fluid 
perturbations are decomposed according to

$$
\begin{aligned}
\delta u_{i}^{\text {polar }} & =-e^{\nu} \sum_{l, m}\left(u_{1}^{l m}, u_{2}^{l m} \partial_{\theta}, u_{2}^{l m} \partial_{\phi}\right) Y_{l m}, \\
\delta u_{i}^{a x i a l} & =-e^{\nu} \sum_{l, m}\left(0,-u_{3}^{l m} \frac{\partial_{\phi}}{\sin \theta}, u_{3}^{l m} \sin \theta \partial_{\theta}\right) Y_{l m}, \\
\delta \epsilon & =\sum_{l, m} \rho^{l m} Y_{l m}, \quad \delta p=(p+\epsilon) \sum_{l, m} H^{l m} Y_{l m}, \\
\xi^{r} & =\left[\nu^{\prime}\left(1-\frac{\Gamma_{1}}{\Gamma}\right)\right]^{-1} \sum_{l, m} \xi^{l m} Y_{l m} .
\end{aligned}
$$

From Eq. (28), we obtain the relation

$$
\rho^{l m}=\frac{(p+\epsilon)^{2}}{\Gamma_{1} p}\left(H^{l m}-\xi^{l m}\right) .
$$

The requirement (33) leads to the following three conditions for the shift components

$$
\begin{aligned}
S_{2}^{l m} & =\frac{1}{2} K_{4}^{l m}-\omega e^{-2 \lambda}\left(\mathrm{i} m V_{3}^{l m}+\mathcal{L}_{1}^{ \pm 1} V_{4}^{l m}\right), \\
V_{1}^{l m} & =K_{5}^{l m}, \\
V_{2}^{l m} & =K_{6}^{l m},
\end{aligned}
$$

where the operator $\mathcal{L}_{1}^{ \pm 1}$ is defined by its action on a perturbation variable $P^{l m}$

$$
\mathcal{L}_{1}^{ \pm 1} P^{l m}=(l-1) Q_{l m} P^{l-1 m}-(l+2) Q_{l+1 m} P^{l+1 m},
$$

with

$$
Q_{l m}:=\sqrt{\frac{(l-m)(l+m)}{(2 l-1)(2 l+1)}} .
$$

These requirements completely specify the gauge, and we obtain three evolution equations for the metric perturbations $S_{3}, V_{3}$ and $V_{4}$, as well as for the six extrinsic curvature components $K_{1}$ to $K_{6}$. From the perturbed energymomentum conservation $\delta\left(T_{; \mu}^{\mu \nu}\right)=0$, we obtain the evolution equations for the enthalpy pertubations $H$ and the velocity perturbations $u_{i}$. In the nonbarotropic case, we can derive the evolution equation for $\xi$ from the relation (28). In addition to the evolution equations, the Einstein equations yield four constraint equations which do not contain any time deriratives. The complete set of equations is rather lengthy and can be found in 82 . 


\subsection{Axial Equations}

We will first focus on the purely axial case. Neglecting the coupling to the polar equations, we have four evolution equations for the metric variable $V_{4}$, the two axial components $K_{3}$ and $K_{6}$ and the axial component of the 4-velocity $u_{3}$ (in the following we omit the indices $l$ and $m$ )

$$
\begin{aligned}
& \left(\frac{\partial}{\partial t}+\mathrm{i} m \omega\right) V_{4}=e^{2 \nu-2 \lambda}\left[K_{6}^{\prime}+\left(\nu^{\prime}-\lambda^{\prime}-\frac{2}{r}\right) K_{6}-e^{2 \lambda} K_{3}\right], \\
& \left(\frac{\partial}{\partial t}+\mathrm{i} m \omega\right) K_{3}=\frac{l(l+1)-2}{r^{2}} V_{4}+\frac{2 \mathrm{i} m}{l(l+1)} \omega^{\prime} e^{-2 \lambda} K_{6}, \\
& \left(\frac{\partial}{\partial t}+\mathrm{i} m \omega\right) K_{6}=V_{4}^{\prime}-\frac{\mathrm{i} m r^{2}}{l(l+1)}\left[\omega^{\prime} K_{3}-16 \pi(\Omega-\omega)(p+\epsilon) u_{3}\right], \\
& \left(\frac{\partial}{\partial t}+\mathrm{i} m \Omega\right) u_{3}=\frac{2 \mathrm{i} m(\Omega-\omega)}{l(l+1)}\left(u_{3}-K_{6}\right),
\end{aligned}
$$

together with the momentum constraint equation

$$
16 \pi(p+\epsilon) u_{3}=K_{3}^{\prime}+\frac{2}{r} K_{3}-\frac{l(l+1)-2}{r^{2}} K_{6}-\frac{2 \mathrm{i} m \omega^{\prime}}{l(l+1)} e^{-2 \nu} V_{4} .
$$

In the literature, the two axial metric components are normally denoted by $h_{0}$ and $h_{1}$. In the above equations, $V_{4}$ is proportional to $h_{1}$ and $K_{6}$ is proportional to $h_{0}$. In the non-rotating case $\dot{u}_{3}=0$, and the remaining equations (49) - (51) are equivalent to a single wave equation for $V_{4}$, which in the exterior reduces to the well-known Regge-Wheeler equation. For neutron stars, these equations describe the axial $w$-modes, which have been first studied by Chandrasekhar and Ferrari 83 for ultra-relativistic models, where they become long lived "trapped modes", and by Kokkotas $\$$ for less compact models.

In the rotating case, fluid motion is possible, and a new family of modes is expected to appear, the unstable $r$-modes. For ultra-compact and rapidly rotating stars, an ergoregion can appear, which is able to drive some of the $w$-modes unstable. This was known only for scalar and electromagnetic perturbations 85 and has been investigated in detail by Commins and Schut 86

for scalar fields on a stellar background. In the following we will discuss it for the gravitational perturbations themselves.

\subsection{The w-mode instability}

For modes mainly associated with fluid motion, it is the rotation of the star itself which is responsible for dragging a counter-rotating mode forward. For $w$-modes, which are predominantly spacetime oscillations it is not the stellar 
rotation itself, but the associated frame dragging, that influences the behavior of the $w$-modes. In the non-rotating case, the axial $w$-modes do not couple to any fluid oscillations at all. If the star is set into rotation, this remains only approximately true as the fluid motion is induced by Eq. (52), and in principle also through the coupling to the polar equations.

But if one still regards the $w$-mode oscillations as being essentially independent of the fluid, then it becomes clear that the only way to make a $w$-mode unstable is to create an ergosphere, i.e. a region where no timelike counter-rotating geodesics exist any more, or in other words, a region where the dragging is so strong that any timelike backwards moving trajectory gets dragged forward. This is associated with a change in the sign of $g_{t t}$.

To test the existence of an ergosphere we write $g_{t t}$ as 86

$$
g_{t t}=-e^{2 \nu}+\omega^{2} r^{2} \sin ^{2} \theta
$$

and restrict ourselves to the equatorial plane $\theta=\pi / 2$. An ergosphere starts to appear whenever $g_{t t}>0$, or equivalently when

$$
e^{\nu}<r \omega .
$$

Models allowing ergospheres have been constructed in the case of rapidly rotating uniform density models by Butterworth and Ipsere8 and for toroidal polytropic configurations by Komatsu, Eriguchi and Hachisu 88 . It is clear that for any realistic equation of state, it is very unlikely to construct stable stellar models which would exhibit an ergosphere, neyertheless recent results suggest the existense of ultra compact quark stars 89 . Therefore, according to the present observational data and the known types of EOS, one would not expect the ergosphere instability of the $w$-modes to be of any significant astrophysical relevance. The compactness needed for a star to develop ergosphere is extremely high i.e. $R / M \sim 2.26-2.4$. An interesting feature of this instability is that it can set in even for quite low rotation rates. For example for a star with $R / M=2.26$ the first $w$-mode becomes unstable at a rotation rate of $0.19 \Omega_{K}$. For $R / M=2.4$ this occurs for $0.83 \Omega_{K}$. Given that a star is becoming unstable due to the existence of an ergosphere then there is no direct dissipation due to shear or bulk viscosity, since the $w$-modes practically do not couple to the fluid motions! This is a intriguing result since it suggests that the instability will grow and the star will radiate away in gravitatignal waves all its available angular momentum before it becomes stable againl

\subsection{The r-mode instability}

In contrast to the $w$-modes, which are predominantly spacetime oscillations, the $r$-modes are characterized by strong axial fluid currents. Even though, it is

florence: submitted to World Scientific on June 8, 2018 


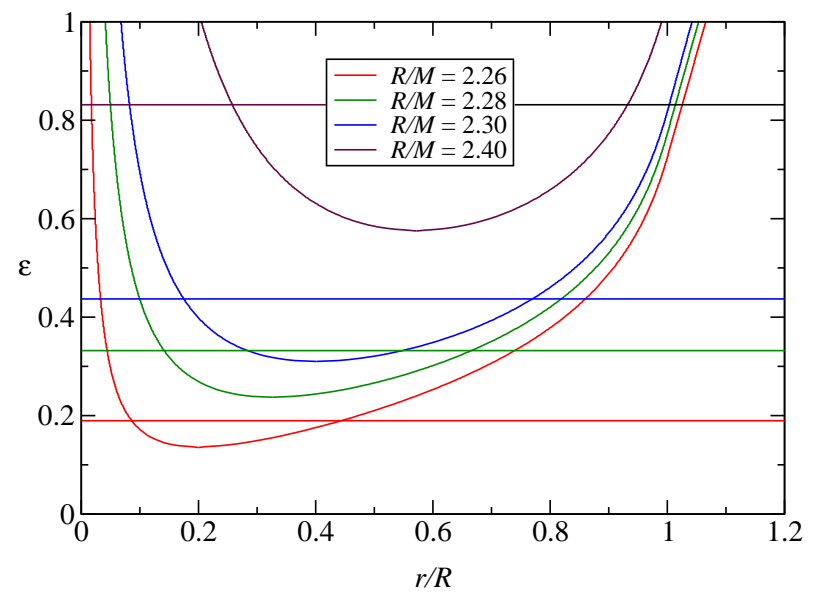

Figure 5. Appearance of the ergoregion as a function of the rotation rate $\varepsilon=\Omega / \Omega_{K}$. For the stellar model with $R / M=2.4$, it first appears at $r=0.2 R$ for $\varepsilon=0.15$ and expands as $\varepsilon$ is increased. The vertical lines represent the rotation rates, at which the first $w$-mode of the respective models becomes unstable.

only through the metric perturbations, that the $r$-modes are driven unstable, it is instructive to study their features in various approximations, where some or all metric perturbations are neglected.

The most radical way is to neglect all metric perturbations. For modes with small damping, such as the polar $p$-modes for example, this so-called relativistic Cowling approximation yields quite accurate results. In Newtonian theory, it also yields very reliable results for the $r$-modes. Let us therefore apply it to the relativistic case as well. Neglecting all metric perturbation in our set of axial evolution equations (49) - (52), the only remaining equation is that for the fluid variable $u_{3}$, which can be written as

$$
\frac{\partial}{\partial t} u_{3}=-i m\left(\Omega-\frac{2 \varpi}{l(l+1)}\right) u_{3} .
$$

From this equation we can immediately deduce that the fluid layers are decoupled from each other, which means that each layer has its own real oscillation frequency given by

$$
\sigma=-m\left(\Omega-\frac{2 \varpi}{l(l+1)}\right)
$$




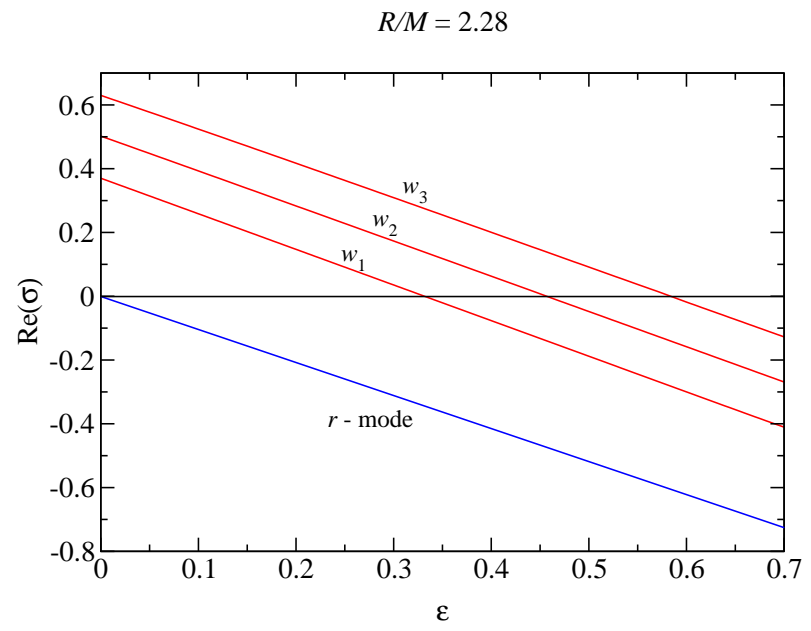

Figure 6 . The real part of the frequency of the three $w$-mode together with the $r$-mode of the stellar model with $R / M=2.28$. The $w_{1}$-mode crosses the zero line at $\varepsilon=0.32$.

The whole frequency range is determined by the values of $\varpi$ at the center and at the stellar surface, and is the larger the more relativistic the stellar model is. In the Newtonian limit $(\varpi \rightarrow \Omega)$, Eq. (57) yields the well known $r$-mode frequency

$$
\sigma_{\mathrm{N}}=-m \Omega\left(1-\frac{2}{l(l+1)}\right)
$$

In the relativistic case, the presence of the frame dragging $\omega$ destroys the occurrence of a single mode frequency and gives rise to a continuous spectrum, at least to this order of approximation.

This somewhat peculiar result might suggest that the Cowling approximation fails in the relativistic case, and we should rather keep some of the metric perturbations. To assess which ones to keep, we consider the nonrotating case. There $\dot{u}_{3}=0$, i.e. we have no oscillatory motion. We can have, however, have a stationary fluid current with a nonzero associated metric perturbation, which we can easily find by setting in our evolution equations (49) - (52) all the time derivatives to zero (together with the rotational terms). From equations (50) and (51), we immediately see that $V_{4}$ has to vanish and 
from Eq. 49, we obtain

$$
K_{6}^{\prime}+\left(\nu^{\prime}-\lambda^{\prime}-\frac{2}{r}\right) K_{6}-e^{2 \lambda} K_{3}=0 .
$$

Moreover, we have the constraint (53), which we can combine with the above equation to yield a single equation for $K_{6}$. To this end, we differentiate (59) with respect to $r$ and use the constraint (53) to eliminate $K_{3}$. This gives us an elliptic second order equation for $K_{6}$ with a source term containing the fluid velocity $u_{3}$. Instead of using $K_{6}$, it is more convenient to write this equation in terms of the variable $h_{0}=e^{\nu-\lambda} K_{6}$

$e^{-2 \lambda} h_{0}^{\prime \prime}-4 \pi r(p+\epsilon) h_{0}^{\prime}+\left[8 \pi(p+\epsilon)+\frac{4 M}{r^{3}}-\frac{l(l+1)}{r^{2}}\right] h_{0}=16 \pi e^{\nu-\lambda}(p+\epsilon) u_{3}$.

Although this equation is derived under the assumption of stationary perturbations, we can also use it for slowly rotating starts and low-frequency oscillations. In this case we no longer assume $\dot{u}_{3}=0$, but we use the evolution equation (52) to govern the time dependence of $u_{3}$.

It is obvious that the above equation (60) together with the evolution equation (52) is qualitatively different from the Cowling approximation (56). In the latter case, the fluid layers are completely decoupled and each one oscillates with its own frequency. With the inclusion of $h_{0}$, we have an elliptic Poisson-like equation, with the fluid acting as a source. As $h_{0}$ couples back into the evolution equation (52), it connects the fluid cells and might give rise to some coherent motion.

To find out whether we can find mode solutions, we assume a harmonic time dependence of $u_{3}$, i.e. $u_{3} \sim \exp (\mathrm{i} \sigma t)$. With this ansatz, we can combine equations (52) and (60) into a single equation, which can be written as

$$
\begin{gathered}
(\alpha-\hat{\varpi})\left[e^{-2 \lambda} h_{0}^{\prime \prime}-4 \pi r(p+\epsilon) h_{0}^{\prime}-\left(8 \pi(p+\epsilon)-\frac{4 M}{r^{3}}+\frac{l(l+1)}{r^{2}}\right) h_{0}\right] \\
+16 \pi(p+\epsilon) \alpha h_{0}=0,
\end{gathered}
$$

where we have introduced the normalized frequency

$$
\alpha=\frac{1}{2} l(l+1)\left(1+\frac{\sigma}{m \Omega}\right)
$$

and

$$
\hat{\varpi}:=\varpi / \Omega .
$$

The relation between $u_{3}$ and $h_{0}$ becomes

$$
u_{3}=\frac{\hat{\varpi}}{\hat{\varpi}-\alpha} e^{\lambda-\nu} h_{0} .
$$




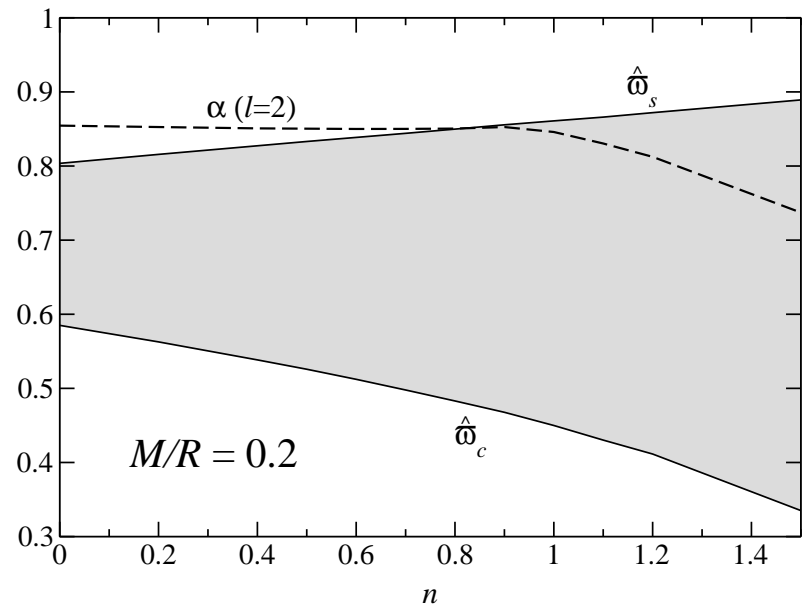

Figure 7. The boundaries of the continuous spectrum $\hat{\varpi}_{c}$ and $\hat{\varpi}_{s}$ together with the $r$-mode frequency $\alpha$ as a function of the polytropic index $n$ for stellar models with compactness of $M / R=0.2$. The case $n=0$ corresponds to a uniform density model.

Eq. (61), which is Kojima's master equation69, is a singular eigenvalue problem, because the factor $\alpha-\hat{\varpi}$ becomes zero if $\alpha$ falls into the range

$$
\hat{\varpi}_{c}<\alpha<\hat{\varpi}_{s} \text {. }
$$

In this case, the fluid perturbation diverges unless $h_{0}$ vanishes at the singular point, which one can prove not to be the case 2 . If we translate the singular range for $\alpha$ back into the corresponding range for the eigenfrequency $\sigma$, we see that it is exactly the same as the continuous frequency spectrum we found in the Cowling approximation given by Eq. (57).

One can show that mode solutions can only exist for $\alpha \leq 171$. If $\alpha$ lies in the range $\hat{\varpi}_{s}<\alpha \leq 1$, the eigenvalue equation is no longer singular, as the singular point now lies outside the star, where $u_{3}$ is zero. Only for such values of $\alpha$, regular mode solutions can be found.

For uniform density models one can indeed find regular mode solutions 71 . However for polytropic equationg gf state, this is only possible for a very restricted range of stellar models 72.73 . The existence of regular mode solutions strongly depends on the polytropic index $n$ and the compactness of the stellar 


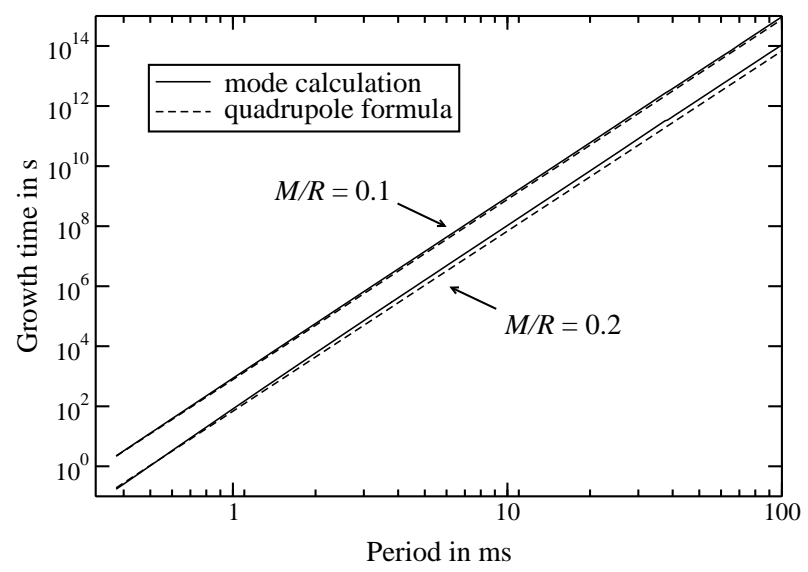

Figure 8. Double logarithmic plot of the growth time for uniform density models with compactness $M / R=0.1,0.2$. Also included is the growth time deduced from formula (70), which agree well with the numerical results.

model under consideration. The general picture is that the smaller $n$ is, the larger is the compactness range where one can find physical mode solutions. For a given polytropic index $n$, one usually finds physical mode solutions for models with a small $M / R$ ratio. As the compactness is increased, i.e. as the models become more relativistic, the mode frequency $\alpha$ decreases and starts approaching $\hat{\varpi}_{s}$. Eventually it crosses this point and migrates inside the range of the continuous spectrum, thus becoming unphysical, and no $r$-mode exists any more. This is shown in Fig. ㄱ.

It has been argued that the singular structure which is associated with the continuous spectrum is an artefact of neglecting the radiation reaction. Both in the Cowling and low-frequency approximations, the eigenfrequency $\sigma$ is always real, whereas it actually should be complex since the emission of gravitational waves should either damp the modes or make them grow. If the frequency is complex, the singular point is removed from the real axis, and the differential equation is no longer singular.

That the inclusion of the radiation reaction does not resolve this issue has been shown by Ruoff and Kokkotas 67 by solving the full set of axial equations 
and by Yoshida and Futamase 4 by imposing a near-zone boundary condition for Kojima's equations instead of the asymptotic flatness condition.

The conversion of the evolution system (49) - (52) into a time independent form is straightforward. Assuming a harmonic time dependence $e^{i \sigma t}$, we can deduce the following two ordinary differential equations

$$
\begin{aligned}
& V_{4}^{\prime}=\frac{\mathrm{i} m r^{2}}{l(l+1)}\left(\omega^{\prime} K_{3}-16 \pi \varpi(p+\epsilon) u_{3}\right)+\mathrm{i}(\sigma+m \omega) K_{6}, \\
& K_{6}^{\prime}=e^{2 \lambda} K_{3}-\left(\nu^{\prime}-\lambda^{\prime}-\frac{2}{r}\right) K_{6}+\mathrm{i} e^{2 \lambda-2 \nu}(\sigma+m \omega) V_{4},
\end{aligned}
$$

together with the two algebraic relations

$$
\begin{aligned}
K_{3} & =\frac{-\mathrm{i}}{\sigma+m \omega}\left(\frac{l(l+1)-2}{r^{2}} V_{4}+\frac{2 \mathrm{i} m \omega^{\prime}}{l(l+1)} e^{-2 \lambda} K_{6}\right), \\
u_{3} & =\frac{2 m \varpi}{2 m \varpi-l(l+1)(\sigma+m \Omega)} K_{6} .
\end{aligned}
$$

Again, Eq. (69) becomes singular if $\sigma$ is real and falls in the range of the continuous spectrum. For real $\sigma$ the denominator $\sigma+m \omega$ in Eq. (68) can also vanish and the equation becomes ill defined. However, as $\sigma$ is expected to be a complex frequency, both equations for $K_{3}$ and $u_{3}$ should remain regular.

For uniform density models, the low-frequency approximation always yielded $r$-mode solutions. The inclusion of the radiation reaction should then yield the associated growth times. For stellar models, which are not too compact, the fully relativistic calculation can indeed reproduce the Newtonian value given for $l=m=2$ by 90

$$
\tau_{\mathrm{PN}}=22\left(\frac{1.4 M_{\odot}}{M}\right)\left(\frac{10 \mathrm{~km}}{R}\right)^{4}\left(\frac{P}{1 \mathrm{~ms}}\right)^{6} \mathrm{~s}
$$

with a remarkably good agreement. For very compact models, however, the growth times found in the fully relativistic case are in general larger than suggested by formula (70). In Fig. 8 we show the growth times for two uniform density models with $M / R=0.1$ and 0.2 as function of the rotation period.

When switching to polytropes, we still find the same pictures as in the low-frequency approximation. As long as the mode is well outside the continuous spectrum, we find a non-vanishing imaginary part, but as soon as the mode approaches the continuous spectrum, the imaginary part starts to decrease and drops to zero at the point where the real part enters the continuous spectrum. This is depicted in Fig. 9, where the dependence of the complex eigenfrequency $\sigma$ on the polytropic index $n$ is shown. For small $n$, the real part of $\sigma$ lies well outside the continuous spectrum, but as $n$ increases, the 


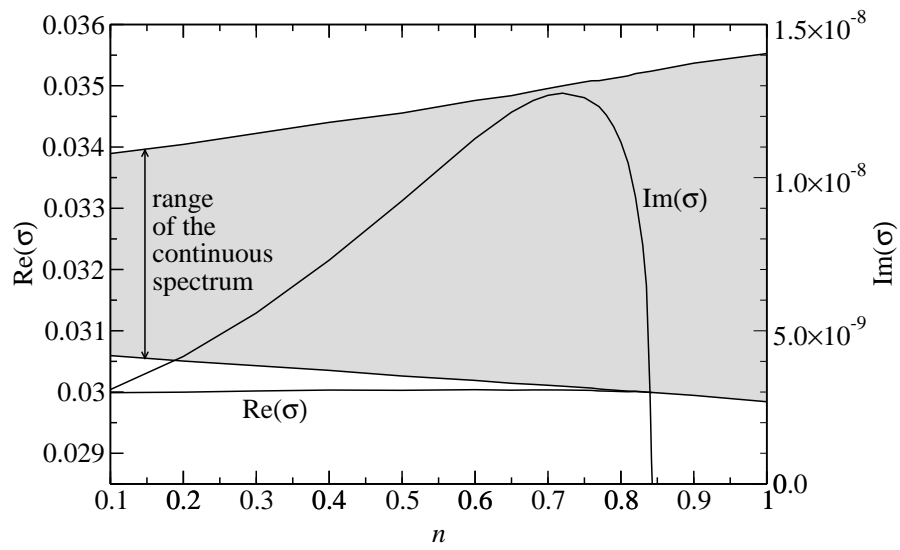

Figure 9. Behavior of the real and imaginary part of the $r$-mode frequency $\sigma$ as a function of the polytropic index $n$ for models with $M / R=0.2$ and rotation period of $1.0 \mathrm{~ms}$. The scale on the left is for the real part, the scale on the right for the imaginary part of $\sigma$. For small $n$, the real part $\operatorname{Re}(\sigma)$ lies outside the range of the continuous spectrum. For increasing $n$, $\operatorname{Re}(\sigma)$ approaches the lower boundary and the imaginary part $\operatorname{Im}(\sigma)$ eventually starts to decrease. For $n=0.843, \Re(\sigma)$ reaches the lower boundary of the continuous spectrum and $\operatorname{Im}(\sigma)$ goes to zero. For larger $n$, no mode can be found any more.

continuous spectrum expands and approaches the real part of $\sigma$. The imaginary part first grows with $n$, but as the continuous spectrum expands and approaches the real part of $\sigma$, it bends down and goes to zero very rapidly. For $n=0.843$, is becomes zero and for larger $n$, a regular mode no longer exists.

\subsection{Inclusion of the coupling}

The above investigations have shown that purely axial modes do not necessarily exist in relativistic non-barotropic stars. So far we have assumed that the coupling to the polar equations can be neglected. However, as the results of the previous sections have shown, this might lead to very unexpected results. To assess, whether the existence of the $r$-modes indeed depends on the stellar model, one has to go one step further and include the coupling to the polar equations. As the complete set of equations is quite complicated, it is useful to study the effect of the coupling within some further approximations.

Although the Cowling approximation did not give any useful results in 
the purely axial case, it nevertheless yields the key to solving the puzzling results of the previous sections.

The first order evolution equations in the Cowling approximation are derived from the perturbed energy-momentum conservation law $\delta\left(T_{; \mu}^{\mu \nu}\right)=0$, and read in the non-barotropic case 2 .

$$
\begin{aligned}
& \left(\partial_{t}+\mathrm{i} m \Omega\right) H=e^{2 \nu-2 \lambda}\left\{C _ { s } ^ { 2 } \left[u_{1}^{\prime}+\left(2 \nu^{\prime}-\lambda^{\prime}+\frac{2}{r}\right) u_{1}-e^{2 \lambda} \frac{l(l+1)}{r^{2}} u_{2}\right.\right. \\
& \left.\left.+2 \mathrm{i} m \varpi e^{2 \lambda-2 \nu} H\right]-\nu^{\prime} u_{1}\right\} \text {, } \\
& \left(\partial_{t}+\mathrm{i} m \Omega\right) u_{1}=H^{\prime}+\frac{p^{\prime}}{\Gamma_{1} p}\left[\left(\frac{\Gamma_{1}}{\Gamma}-1\right) H+\xi\right]-B\left(\mathrm{i} m u_{2}+\mathcal{L}_{1}^{ \pm 1} u_{3}\right), \\
& \left(\partial_{t}+\mathrm{i} m \Omega\right) u_{2}=H+\frac{2 \varpi}{l(l+1)}\left(\mathrm{i} m u_{2}+\mathcal{L}_{3}^{ \pm 1} u_{3}\right)-\mathrm{i} m \frac{r^{2} e^{-2 \lambda}}{l(l+1)} B u_{1}, \\
& \left(\partial_{t}+\mathrm{i} m \Omega\right) u_{3}=\frac{2 \varpi}{l(l+1)}\left(\mathrm{i} m u_{3}-\mathcal{L}_{3}^{ \pm 1} u_{2}\right)+\frac{r^{2} e^{-2 \lambda}}{l(l+1)} B \mathcal{L}_{2}^{ \pm 1} u_{1}, \\
& \left(\partial_{t}+\mathrm{i} m \Omega\right) \xi=\nu^{\prime}\left(\frac{\Gamma_{1}}{\Gamma}-1\right) e^{2 \nu-2 \lambda} u_{1},
\end{aligned}
$$

with

$$
B=\omega^{\prime}+2 \varpi\left(\nu^{\prime}-\frac{1}{r}\right),
$$

and the coupling operators

$$
\begin{aligned}
& \mathcal{L}_{1}^{ \pm 1} P^{l m}=(l-1) Q_{l m} P^{l-1 m}-(l+2) Q_{l+1 m} P^{l+1 m} \\
& \mathcal{L}_{2}^{ \pm 1} P^{l m}=-(l+1) Q_{l m} P^{l-1 m}+l Q_{l+1 m} P^{l+1 m} \\
& \mathcal{L}_{3}^{ \pm 1} P^{l m}=(l-1)(l+1) Q_{l m} P^{l-1 m}+l(l+2) Q_{l+1 m} P^{l+1 m}
\end{aligned}
$$

As these operators couple equations of order $l$ to equations of order $l \pm 1$, the above set of equations represents a coupled system with $l$ ranging from $l=m$ to $l=\infty$.

\subsection{The Continuous Spectrum}

By assuming a harmonic time dependence, one can easily transform the above time dependent equations into an eigenvalue problem for the frequency $\sigma$. Defining

$$
u^{l}=\left(\begin{array}{c}
u_{2}^{l} \\
u_{3}^{l}
\end{array}\right), \quad s^{l}=\left(\begin{array}{c}
H^{l} \\
u_{1}^{l}
\end{array}\right), \quad l=m, \ldots, \infty
$$


the eigenvalue equations can write be written as a set of ODEs

$$
\left(s^{l}\right)^{\prime}=A s^{l}+B_{-}^{l} u^{l-1}+B^{l} u^{l}+B_{+}^{l} u^{l+1} \quad l=m, \ldots, \infty
$$

together with the algebraic relations

$$
U_{-}^{l} u^{l-1}+U_{\Sigma}^{l} u^{l}+U_{+}^{l} u^{l+1}=S_{-}^{l} s^{l-1}+S^{l} s^{l}+S_{+}^{l} s^{l+1}, \quad l=m, \ldots, \infty,
$$

where the letters $A, B, U$ and $S$ with their various indices represent $2 \times 2$ matrices. The algebraic relations can be viewed as a matrix equation for two infinitely dimensional matrices $\mathrm{U}$ and $\mathrm{S}$ acting on the vectors

$$
\begin{aligned}
& u=\left(u^{m}, u^{m+1}, u^{m+2}, \ldots, u^{l}, \ldots\right)^{\mathrm{T}}, \\
& s=\left(s^{m}, s^{m+1}, s^{m+2}, \ldots, s^{l}, \ldots\right)^{\mathrm{T}},
\end{aligned}
$$

whose respective elements are the 2-vectors $u^{l}$ and $s^{l}$. Both $\mathrm{U}$ and $\mathrm{S}$ are tridiagonal block matrices, with each block given by the above $2 \times 2$ matrices. Now we can write Eq. (82) as

$$
\sum_{l^{\prime}} \mathrm{U}^{l l^{\prime}} u^{l^{\prime}}=\sum_{l^{\prime}} \mathrm{S}^{l l^{\prime}} s^{l^{\prime}}, \quad l=m \ldots \infty,
$$

which can be solved for $u^{l}$ by multiplying both sides with $\mathrm{U}^{-1}$

$$
u^{l}=\sum_{l^{\prime} l^{\prime \prime}}\left(\mathrm{U}^{-1}\right)^{l l^{\prime}} \mathrm{S}^{l^{\prime} l^{\prime \prime}} s^{l^{\prime \prime}}, \quad l=m \ldots \infty .
$$

Note that the matrix $U$ is $r$-dependent since its elements $U_{ \pm}^{l}$ contain the function $\varpi$. For $U$ to be invertible its determinant must not vanish. For certain values of $\sigma$, however, this happens. In the Newtonian limit $\varpi \rightarrow \Omega$, the matrix $\mathrm{U}$ becomes independent of $r$, and one can easily show that $\mathrm{U}$ becomes singular only for a discrete set of frequencies $\sigma$. These frequencies represent the solutions of the homogeneous part $\mathrm{U} u=0$ of Eq. (85). In the relativistic case, however, the zeroes of $\operatorname{det} U$ depend on the position inside the star, which means that each single Newtonian frequency will be spread out into a continuous band of frequencies, determined by the values of $\varpi$ at the center and at the surface of the star, which we will denote by $\varpi_{0}$ and $\varpi_{R}$, respectively. In order to numerically perform the inversion and solve equation (86), we have to truncate the system at some value $l_{\max } \geq m$. To explicitly compute the ranges of the resulting continuous spectrum we need the matrices $U_{-}^{l}, U_{+}^{l}$ and $U_{\Sigma}^{l}$, which are given by

$$
U_{-}^{l}=-2 \hat{\omega} \frac{l-1}{l} Q_{l m}\left(\begin{array}{ll}
0 & 1 \\
1 & 0
\end{array}\right),
$$




$$
\begin{aligned}
& U_{\Sigma}^{l}=\Sigma\left(\begin{array}{ll}
1 & 0 \\
0 & 1
\end{array}\right), \\
& U_{+}^{l}=-2 \hat{\varpi} \frac{l+2}{l+1} Q_{l+1 m}\left(\begin{array}{ll}
0 & 1 \\
1 & 0
\end{array}\right),
\end{aligned}
$$

with

$$
\Sigma=\kappa-\frac{2 m \varpi}{l(l+1)}
$$

and

$$
\kappa=\sigma+m \Omega .
$$

In the following examples, we choose $m=2$. Let us start with the simplest case which is choosing $l_{\max }=2$. Then $\mathrm{U}=U_{\Sigma}^{2}$ and $\operatorname{det} \mathrm{U}=0$ just yields

$$
\Sigma=0,
$$

which gives exactly the same continuous spectrum as following from Eq. (57). For $l_{\max }=3$

$$
\mathrm{U}=\left(\begin{array}{cc}
U_{\Sigma}^{2} & U_{+}^{2} \\
U_{-}^{3} & U_{\Sigma}^{3}
\end{array}\right),
$$

and $\operatorname{det} \mathrm{U}=0$ yields the two solutions

$$
\kappa=\left(\frac{1}{2} \pm \frac{\sqrt{105}}{14}\right) \varpi .
$$

Hence, the two ranges of the continuous spectrum are given by

$$
1.2319 \varpi_{0} \leq \kappa \leq 1.2319 \varpi_{R},
$$

for the plus sign and

$$
-0.2319 \varpi_{0} \leq \kappa \leq-0.2319 \varpi_{R},
$$

for the minus sign. For $l_{\max }=4$, we obtain

$$
\frac{\kappa}{\varpi}=\left\{\begin{array}{c}
1.4964 \\
0.4669 \\
-0.7633
\end{array} .\right.
$$

Each increment of $l_{\max }$ leads to an additional range of the continuous spectrum. Depending on the respective values of $\varpi_{0}$ and $\varpi_{R}$, the various ranges might as well overlap. This actually happens for the more relativistic stars since the variation in $\varpi$ is much greater. 

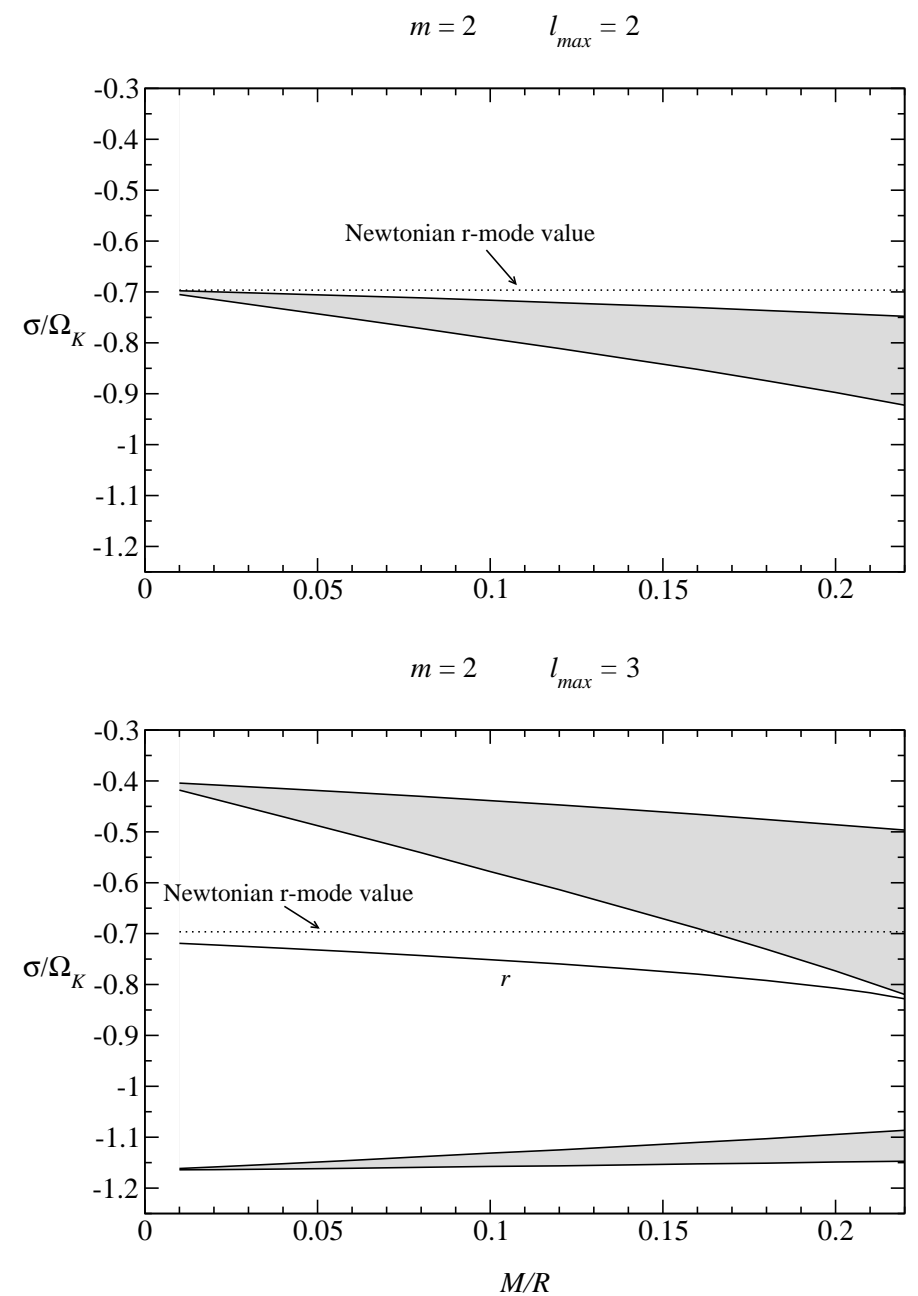

Figure 10. The continuous spectrum and the $r$-mode for $m=2$ as functions of $M / R$ for $l_{\max }=2$ and $l_{\max }=3$. For $l_{\max }=2$, only the continuous spectrum exists. The relativistic $r$-mode appears for $l_{\max }=3$ well outside the continuous spectrum as the latter has shifted away from its previous location for $l_{\max }=2$. The dotted line represent the Newtonian $r$-mode value from Eq. (58).

In the Newtonian limit $\varpi \rightarrow \Omega$, the continuous spectrum shrinks down to discrete values. Interestingly, these values $\kappa / \Omega$, following from $\operatorname{det} U=0$, 

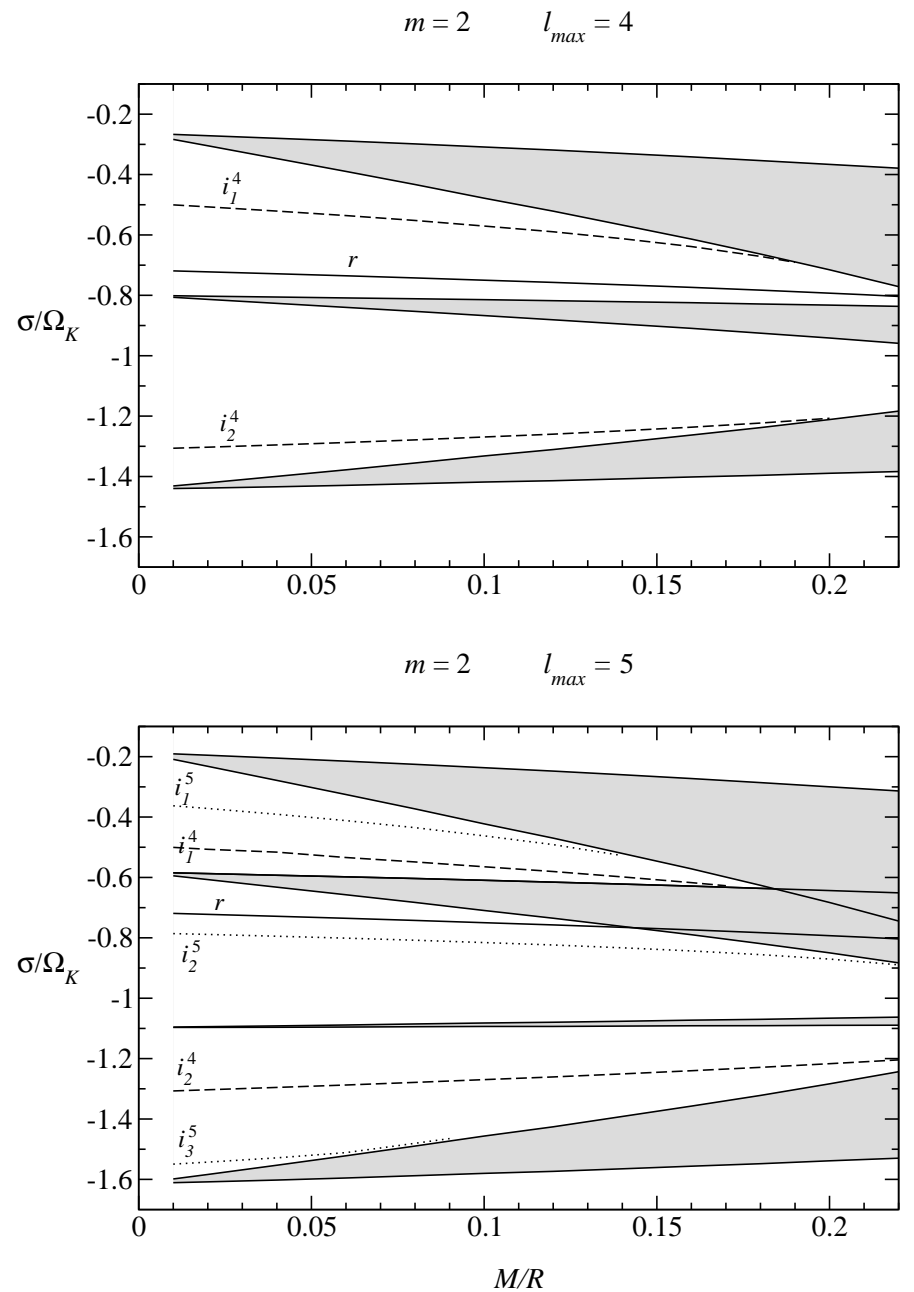

Figure 11. Same as in Fig. 10 for $m=2$. In addition we have plotted the Newtonian value for the $r$-mode according to Formula (58). The relativistic $r$-mode appears for $l_{\max }=3$ well outside the continuous spectrum as the latter has shifted away from its previous location for $l_{\max }=2$. Only the $r$-mode and the $i_{2}^{4}$ and $i_{2}^{5}$ modes can exist for the whole compactness range.

are exactly the eigenvalues $\kappa_{0}$ of the (generalized) $r$-modes of the Maclaurin spheroids in the limit of low angular velocities, which were computed by 
Lindblom and Ipser 91 and presented in their Table 1. Our above values have been obtained for $m=2$, but we can reproduce all their values for $m=0$ to $m=3$. Note, however, that their $l$ is related to our $l_{\max }$ by $l=l_{\max }-1$. Also note that these values are completely independent of any stellar structure parameters.

In Figs. 10 and 11 we show both the continuous spectrum and the eigenmodes as functions of the compactness $M / R$ for different values of $l_{\max }$. The modes are for barptropic stellar models, based on a polytropic equation of state with $n=1$ 1 Starting for $l_{\max }=2$ (upper panel of Fig. 10), we find no mode, but only a band of frequencies given by Eq. (57). We have also included the Newtonian value for the $r$-mode as given by Eq. (58). One can clearly see that in the limit $M / R \rightarrow 0$ the continuous spectrum shrinks to this value. If we include the coupling to the polar equations with $l=3$, the situation changes drastically. As expected we now obtain two continuous frequency bands and a mode which lies below the Newtonian $r$-mode value, the further below the more compact the star is. Translating to positive frequencies, this means that this mode always has a higher frequency than the Newtonian $r$ mode. Inspection of the eigenfunction reveals that the axial part is well described by a $r^{l+1}$ power law, i.e.

$$
u_{3}^{l=2} \sim r^{3},
$$

which is exactly what one expects for the $r$-mode. Inclusion of higher $l$ only gives minor frequency corrections, which tells us that this is indeed a physical inertial mode, the relativistic version of an $m=2 r$-mode. According to our nomenclature for inertial modes, the $r$-mode is actually an $i_{1}^{3}$ inertial mode. In Fig. 11, we have also included the coupling up to $l_{\max }=4$ and 5. Here, each inclusion of one additional $l$ leads to another patch in the continuous spectrum, and to the appearance of some new inertial modes. Notice that the width of the uppermost and lowermost patches is very broad, whereas the middle patches around $\sigma / \Omega_{K} \approx-1$ remain very narrow, even for large $M / R$. This is because the width of a patch is given by $\kappa\left(\varpi_{R}-\varpi_{0}\right)$, which is therefore the smaller the closer $\kappa$ is to zero. For any given $l_{\max }$, we find $l_{\max }-m$ new inertial modes. We label them $i_{n}^{l}$ with $l=l_{\max }$ and $n=1, \ldots, l-m$. In the Newtonian limit, they appear very close to the values found from $\operatorname{det} \mathrm{U}=0$ for $l_{\max }-1$. This is what to be expected from the Newtonian theory of inertial modes, since the eigenfrequency can be computed at lower order than the eigenfunction. This means that for the inertial modes $i_{n}^{l}$ appearing at $l_{\max }=l$, the associated eigenfrequencies can already be inferred from the solutions of $\operatorname{det} \mathrm{U}=0$ for $l_{\max }-1$.

From Fig. 10, it is clear that the $r$-mode exists for the whole compactness

florence: submitted to World Scientific on June 8, 2018 
range, as it is always located outside the continuous spectrum. For $l_{\max }=4$ (upper panel of Fig. 11), it is still outside the continuous spectrum, but for $l_{\max }=5$ (lower panel of Fig. 11), it lies inside the continuous spectrum for $M / R>0.14$. In this case, the eigenvalue equations cannot be solved any more, as $\mathrm{U}$ becomes singular. It is only through the evolution of the time dependent equations that one can see that the $r$-mode exists inside the continuous spectrum. This is not only the case for the $r$-mode, but also for other inertial modes. However, not all the modes are able to survive inside the continuous spectrum. This can be seen for the inertial modes $i_{1}^{4}$ and $i_{2}^{4}$ in Fig. 11. For $l_{\max }=4$, they both cease to exist when they reach the continuous spectrum. For $l_{\max }=5$ the continuous spectrum has shifted such that the $i_{2}^{4}$ mode remains always outside whereas the $i_{1}^{4}$ mode still cannot penetrate the continuous spectrum. For larger $l_{\max }$, one can find the $i_{2}^{4}$ mode inside the continuous spectrum, but not the $i_{1}^{4}$ mode.

These results can be summarized as follows. As a result of the relativistic frame dragging, there always exists a continuous spectrum, which, however, depends very strongly on the number of coupled equations. For $l_{\max }=2$, the continuous spectrum is confined to a single connected region, with increasing width for more relativistic stellar models. If $l_{\max }$ is increased the continuous spectrum splits into an increasing number of patches, which are disconnected for weakly relativistic stars but tend to overlap for more compact stellar models. In the limit $l_{\max } \rightarrow \infty$ the continuous spectrum should completely cover the frequency range of the inertial modes. Nevertheless, there are inertial modes, in particular the $r$-mode, which can exist inside the continuous spectrum. For very relativistic stars, however, the continuous spectrum is able to destroy some of the inertial modes.

Caution should be used in order to draw any conclusions about the (non)existence of these modes for a rapidly rotating neutron star. The above results showed that the continuous spectrum is very sensitive to the number of equations that are coupled. So far, only first order rotational corrections have been taken into account, i.e. only the coupling from $l$ to $l \pm 1$. Inclusion of higher order corrections leads to coupling to higher $l$, which can modify the continuous spectrum in a significant way. It is therefore possible that higher order corrections affect the continuous spectrum in such a way that modes which do not exist in the first order analysis can now exist because the responsible patch could have moved elsewhere.

Of course, another important step consists in including the metric perturbations. Lockitch et al.71 included some (non-radiative) metric perturbations, but restricted their studies to a post Newtonian treatment. Still, the continuous spectrum will remain unaffected when metric variables are included, for it 
is only the fluid equations which are responsible for its existence. However, by including the metric perturbations, the modes might be affected in such a way that they get pushed out of the continuous spectrum and we might find some of the modes which we cannot find in the Cowling approximation. That this might true can be seen from the purely axial $l=m$ case, which we have discussed in the previous sections. There, the Cowling approximation leads to a purely continuous spectrum without any mode solutions. In the low-frequency approximation, where one retains a certain metric component, one is lead to Kojima's master equation, which in certain cases admits $r$-mode solutions, whose frequencies lie outside the continuous spectrum. But the range of the continuous spectrum is still the same as in the Cowling approximation and even when all the metric perturbations were included, the qualitative picture remained the same.

Nevertheless it is clear that the relativistic case is quite different from the Newtonian one, and further studies are necessary to gain a full understanding of the inertial modes of (rapidly) rotating relativistic stars.

\section{Acknowledgments}

We are grateful to N. Andersson, E. Berti, U. Sperhake, N. Stergioulas, A. Stavridis and M.Vavoulidis for many useful comments and suggestions. This work has been supported by the EU Programme 'Improving the Human Research Potential and the Socio-Economic Knowledge Base' (Research Training Network Contract HPRN-CT-2000-00137). J.R. is supported by the Marie Curie Fellowship No. HPMF-CT-1999-00364.

\section{References}

1. N. Andersson Astrophys. J., 502, 708 (1998)

2. J. L. Friedman and S. M. Morsink Astrophys. J., 502, 714 (1998)

3. S. Chandrasekhar Phys. Rev. Lett., 24, 611 (1970a)

4. S. Chandrasekhar Astrophys. J., 161, 561 (1970b)

5. J. L. Friedman and B. F. Schutz Astrophys. J., 221, 937 (1978)

6. J. L. Friedman and B. F. Schutz Astrophys. J., 222, 281 (1978)

7. N. Stergioulas Living Rev. Rel. 1, 8 (1998)

8. N. Andersson and K. D. Kokkotas I.J.M.P. D 10, 381 (2001)

9. K. D. Kokkotas, J. Ruoff and N. Andersson gr-qc/0212429

10. S. Chandrasekhar Astrophys. J., 140, 417 (1964)

11. K. D. Kokkotas and J. Ruoff A\&A 366, 565 (2001) 
12. W. Unno, Y. Osaki, H. Ando and H. Shibahashi Nonradial oscillations of stars (University of Tokyo Press, 1989)

13. K. S. Thorne and A. Campolattaro Astrophys. J., 149, 591 (1967); Erratum Astrophys. J., 152, 673 (1968)

14. H. Poincaré Figrues d'Equilibre d'une Masse Fluide (C.Naud, Paris) (1903)

15. P. Haensel and J.L.Zdunik Nature 340, 617 (1989)

16. P. Haensel, M. Salgado, S. Bonazzola A\&A 296, 746 (1995)

17. J. L. Friedman, J. R. Ipser, R. D. Sorkin Astrophys. J., 325, 722 (1988)

18. J.L.Houser, J.M.Centrella, S.C.Smith Phys. Rev. Lett., 72, 1314 (1994)

19. S.C.Smith, J.L.Houser, J.M.Centrella Astrophys. J., 458, 236 (1996)

20. Y.T.Liu Phys. Rev. D, 65, 124003 (2002)

21. S. Bonazzola, J. Frieben, E. Gourgoulhon Astrophys. J., 460, 379 (1996)

22. D. Skinner and L. Lindblom Astrophys. J., 461, 920 (1996)

23. S. L. Detweiler and L. Lindblom Astrophys. J., 213, 193 (1977)

24. J.L. Friedman, Commun. Math. Phys. 62247 (1978)

25. J. R. Ipser, L. Lindblom Astrophys. J., 373, 213 (1991)

26. R. F. Sawer Phys. Rev. D, 39, 3804 (1989)

27. C. Cutler, L. Lindblom Astrophys. J., 385, 630 (1992)

28. K. S. Thorne Rev. Mod. Phys.52, 299 (1980)

29. L. Lindblom, B. J. Owen and S. M. Morsink Phys. Rev. Lett. , 80, 4843 (1998)

30. N. Andersson, K. D. Kokkotas and B. F. Schutz Astrophys. J., 510, 846 (1999)

31. L. Bildsten and G. Ushomirsky Astrophys. J. Lett., 529, 33 (2000)

32. L. Lindblom, B. J. Owen and G. Ushomirsky Phys. Rev. D, 62, 084030 (2000)

33. S. Yoshida and U. Lee Astrophys. J., 546, 1121 (2001)

34. Y. Levin and G. Ushomirsky Mon. Not. R. Astr. Soc., 324, 917 (2001)

35. J. Madsen Phys. Rev. Lett., 81, 3311 (1998)

36. N. Andersson, D. I. Jones, K. D. Kokkotas Mon. Not. R. Astr. Soc., 337, $1224(2002)$

37. P.B.Jones Phys. Rev. Lett., 86, 1384 (2001)

38. P.B.Jones Phys. Rev. D, 64, 084003 (2001)

39. L.Lindblom and B.J.Owen Phys. Rev. D, 65, 063006 (2002)

40. B. J. Owen, L. Lindblom, C. Cutler, B. F. Schutz, A. Veccio and N. Andersson Phys. Rev. D, 58, 084020 (1998)

41. P. R. Brady and T. Greighton Phys. Rev. D, 61, 082001 (2000)

42. B. J. Owen and L. Lindblom Class. Quantum Grav., 19, 1247 (2002)

43. J. Papaloizou and J. E. Pringle Mon. Not. R. Astr. Soc., 182, 423 
(1978)

44. R.V. Wagoner Astrophys. J., 278, 345 (1984)

45. N. Andersson, K. D. Kokkotas and N. Stergioulas Astrophys. J., 516, 307 (1999)

46. L. Bildsten Astrophys. J. Lett., 501, 89 (1998)

47. Y. Levin Astrophys. J., 517, 328 (1999)

48. N. Andersson, D. I. Jones, K. D. Kokkotas and N. Stergioulas Astrophys. J. Lett., 534, 75 (2000)

49. H. C. Spruit A\&A 341, L1 (1999)

50. L. Rezzolla, F. K. Lamb and S. L. Shapiro Astrophys. J. Lett., 531, L139 (2000)

51. L. Rezzolla, F. L. Lamb, D. Markovic and S. L. Shapiro Phys. Rev. D, 64, 104013 (2001a)

52. L. Rezzolla, F. L. Lamb, D. Markovic and S. L. Shapiro Phys. Rev. D, 64, 104014 (2001b)

53. W. C. G. Ho and D. Lai Astrophys. J., 543, 386 (2000)

54. S. M. Morsink and V. Rezania Astrophys. J., 574, 908 (2002)

55. N. Stergioulas and J. A. Font Phys. Rev. Lett. , 86, 1148 (2001)

56. L. Lindblom, J. E. Tohline and M. Vallisneri Phys. Rev. Lett. , 86, $1152(2001)$

57. M. Vallisneri see article in this volume

58. A.K.Schenk, P. Arras, E.E.Flanagan, S.A. Teukolsky, I.Wasserman Phys. Rev. D, 65, 024001 (2002)

59. S. M. Morsink Astrophys. J., 571, 435 (2002)

60. P. Arras, E. E. Flanagan, S. M. Morsink, A. K. Schenk, S.A.Teukolsky, I.Wasserman astro-ph/0202345

61. R. V.Wagoner astro-ph/0207589

62. J. B. Hartle, Astrophys. J., 150, 1005 (1967)

63. S. Chandrasekhar, V. Ferrari Proc. R. Soc. London, Ser. A, 433, 423 (1991)

64. Y. Kojima Phys. Rev. D, 46, 4289 (1992)

65. Y. Kojima Astrophys. J., 414, 247 (1993)

66. Y. Kojima Prog. Theor. Phys. 90, 977 (1993)

67. J. Ruoff, K. D. Kokkotas Mon. Not. R. Astr. Soc., 330, 1027 (2002)

68. S. Yoshida, Y. Kojima Mon. Not. R. Astr. Soc., 289, 117 (1997)

69. Y. Kojima Mon. Not. R. Astr. Soc., 293, 49 (1998)

70. H. R. Beyer, K. D. Kokkotas Mon. Not. R. Astr. Soc., 308, 745 (1999)

71. K. H. Lockitch, N. Andersson, J.L. Friedman Phys. Rev. D, 63, 024019 (2001)

72. J. Ruoff, K. D. Kokkotas Mon. Not. R. Astr. Soc., 328, 678 (2001)

florence: submitted to World Scientific on June 8, 2018 
73. S. Yoshida Astrophys. J., 558, 263 (2001)

74. S. Yoshida, T. Futamase Phys. Rev. D, 64, 123001 (2001)

75. Y. Kojima, M. Hosonuma Phys. Rev. D, 62, 044006 (2000)

76. K. H. Lockitch, N. Andersson, gr-qc/0106088

77. S. Yoshida, U. Lee Astrophys. J., 567, 1112 (2002)

78. J. Ruoff, A. Stavridis, K. D. Kokkotas, 2002, in press to MNRAS, grqc/0203052

79. P. N. McDermott, H. M. van Horn, C. J. Hansen Astrophys. J., 325, 725 (1988)

80. L. Battiston, P. Cazzola, L. Lucaroni Nuovo Cimento B, 3, 275, 1971

81. Arnowitt R., Deser S., Misner C.W., 1962, in Witten L., ed., Gravitation: An Introduction to Current Research. Wiley, New York, p.227

82. J. Ruoff, A. Stavridis, K. D. Kokkotas, Mon. Not. R. Astr. Soc., 332, $676(2002)$

83. S. Chandrasekhar, V. Ferrari Proc. R. Soc. London, Ser. A, 434, 449 (1991)

84. K. D. Kokkotas, Mon. Not. R. Astr. Soc., 268, 1015 (1994); Erratum Mon. Not. R. Astr. Soc., 277, 1599 (1995)

85. J. L. Friedman Commun. Math. Phys. 63, 243 (1978)

86. N. Commins and B. F. Schutz Proc. R. Soc. London, Ser. A, 364, 211 (1978)

87. E. M. Butterworth, J. R. Ipser Astrophys. J., 204, 200 (1976)

88. H. Komatsu, Y. Eriguchi, I. Hachisu Mon. Not. R. Astr. Soc., 239, 153 (1989)

89. J. J. Drake et al. astro-ph/0204159

90. K. D. Kokkotas and N. Stergioulas A\&A 341, 110 (1999)

91. L. Lindblom, J. R. Ipser Phys. Rev. D, 59, 044009 (1999) 